\title{
Dianas quirúrgicas en el tratamiento de enfermedades psiquiátricas. Desde el movimiento a las emociones
}

\author{
J. Guridi y G. Aldave
}

Servicio de Neurocirugía. Clínica Universidad de Navarra. Pamplona. España.

\section{Resumen}

La estimulación cerebral profunda para las enfermedades psiquiátricas que son refractarias a los distintos tratamientos convencionales, se está realizando actualmente debido en gran parte a los conocimientos adquiridos en la cirugía para los trastornos del movimiento, sobre todo la enfermedad de Parkinson (EP). La depresión, los trastornos obsesivo-compulsivos (TOC) y el síndrome de Gilles de la Tourette son problemas córtico-estriato-tálamo-corticales relacionados con los circuitos límbicos de los ganglios basales.

En esta revisión se analizan las distintas dianas quirúrgicas para las diferentes patologías neuro-psiquiátricas. Para el TOC actualmente existen dos dianas, el complejo estriado ventral (VS)-núcleo accumbens (Nacc) y el núcleo subtalámico (NST). Para la depresión refractaria, el área subgenual (área 25 de Brodmann) y el VS/Nacc. Para el Tourette, el ventralis oralis interno y centromediano parafascicularis (Voi, CM/Pf) del tálamo y el globo pálido interno (GPi). En la actualidad no existe una única diana específica en cualquiera de las patologías, ya que los resultados clínicos tras la estimulación pueden considerarse similares. Por otro lado, una misma diana quirúrgica puede mejorar diferentes patologías.

PALABRAS CLAVE. Estimulación cerebral profunda. Enfermedad de Parkinson. Trastorno obsesivo-compulsivo. Gilles de la Tourette. Depresión.

Surgical targets in Psychiatric disorders. From movement to emotions

Summary

Deep brain stimulation (DBS) for psychiatric disorders refractory to conventional treatments are currently been performed based in the knowledgment

Recibido: 23-06-10. Aceptado: 5-10-10. obtained in the motor disorder surgery and mainly in Parkinson's disease. Depression, obsessive-compulsive disorder (OCD) and Tourette syndrome, all of them are cortico-striato-thalamo-cortical pathological process involved in the limbic loop of the basal ganglia.

This review describes the different targets in these pathological neuro-psychiatric disorders. For OCD there are currently two targets, ventral striatum (VS) Accumbens nucleus (Nacc) and the subthalamic nucleus (STN). In refractory depression the subgenual area (25 Brodmann area) and VS/Nacc. For Tourette syndrome the ventralis oralis internus and centromedianum/ parafascicularis of the thalamus (Voi and CM/Pf) and the internal part of the globus pallidus (GPi). Currently there are no specific surgical target for each pathological disorder because clinical results reported are very similar after stimulation surgery. In other point, a selected surgical target also may improve different pathologies.

KEY WORDS. Deep brain stimulation. Parkinson's disease. Obsessive-compulsive disorder. Tourette syndrome. Depression.

Introducción

El tratamiento quirúrgico de los problemas psiquiátri-

Abreviaturas. AMS: área motora suplementaria. CA: comisura anterior. rCBF: flujo sanguíneo cerebral regional. CCA: córtex cingular anterior. CI: cápsula interna. CM/Pf: centro medianol parafascicular. COF: córtex orbito-frontal. DA: agonista dopaminérgico. DBS: deep brain stimulation. DM: núcleo dorsomedial. EP: enfermedad de Parkinson. GB: ganglios basales. GPe: globo pálido externo. GPi: globo pálido interno. HDRS: Hamilton Depression Rating Scale. ICD: impulse control disease. Nacc: núcleo accumbens. NST: núcleo subtalámico. PET: tomografía por emisión de positrones. SGT: sindrome Gilles de laTourette. SNc: sustancia negra (pars compacta). SNr: sustancia negra (pars reticulata). TEC: terapia electro-convulsiva. TOC: trastorno obsesivo-compulsivo. Voi: ventral oral interno. VS: estriado ventral. 
cos ha venido realizándose desde los albores de la cirugía con estereotaxia ${ }^{23,105}$. Sin embargo, durante años la cirugía de dichos trastornos dejó de practicarse, en gran parte debido a lo indiscriminado de su realización, lo que llevó a un descrédito de dichas técnicas y a su práctica desaparición. También la aparición de los neurolépticos ha podido beneficiar a muchos pacientes, por lo que la cirugía para los trastornos psiquiátricos se redujo considerablemente. Sin embargo, actualmente con la mejora en técnicas de imagen, disminución de los riesgos quirúrgicos de la cirugía funcional y la aparición de técnicas de estimulación en este campo quirúrgico, todo ello ha conllevado la reintroducción de la cirugía en las enfermedades psiquiátricas refractarias a los diferentes tratamientos convencionales ${ }^{69}$.

La cirugía realizada desde hace varios años en patología de los trastornos del movimiento, tanto enfermedad de Parkinson (EP), distonía como temblor, han llevado a incrementar el conocimiento tanto anatómico como fisiopatológico sobre la organización funcional de los ganglios basales (GB) en los distintos procesos patológicos. Esto en gran medida se debe a los estudios realizados en primates y desde los cuales hemos podido comprender el funcionamiento de los GB en condiciones de hipercinesia e hipocinesia creando un modelo de funcionamiento. Así la lesión del núcleo subtalámico (NST) en animales $\mathrm{y}$ en humanos induce una corea/balismo (estado hipercinético), o bien la introducción de un tóxico como el MPTP (1-metyl-4 fenil-1,2,3,6 tetrahidropiridina) en primates conlleva un cuadro parkinsoniano con temblor, rigidez y lentitud similar a la EP en humanos (condición hipocinética $)^{18,24,27}$. En ambos modelos, el NST juega un papel capital, ya que en el primero, su hipoactividad induciría las disquinesias debido a una hiperactividad del eje tálamo-cortical ${ }^{24}$. En la condición parkinsoniana, sería la hiperactividad del NST la que conllevaría una hiperactividad inhibitoria desde el globo pálido interno (GPi) sobre el tálamo favoreciendo la acinesia en el modelo de $\mathrm{GB}^{18,27}$. De esta manera, la hiperactividad de la proyección NST-GPi es capital para entender el funcionamiento de los GB en el EP. En consecuencia, la lesión tanto por técnicas químicas como por radiofrecuencia, o la inhibición del NST por técnicas de estimulación, conlleva a un beneficio de los animales, tanto del temblor como la rigidez y la lentitud ${ }^{10,16,17,46}$.

La creación de este modelo de funcionamiento por un lado y la aparición de técnicas de estimulación cerebral (deep brain stimulation: DBS) sin tener que lesionar estructuras profundas, así como el buen resultado quirúrgico en este tipo de patología de los trastornos del movimiento sobre todo en la enfermedad de Parkinson, ha llevado a introducir la cirugía de estimulación en los pacientes con trastornos psiquiátricos que son refractarios a las diferentes terapias convencionales. Es por tanto desde la experiencia quirúrgica en los trastornos motores, desde donde partimos para abordar las distintas patologías neuro-psiquiátricas. Además, como veremos en la revisión, ambos campos, están muy relacionados entre sí, ya que las mismas estructuras anatómicas relacionadas con los problemas motores también lo están con las áreas cognitivas y límbicas que regulan las emociones.

La introducción de técnicas de estimulación en el campo de la psiquiatría no es nueva ya que los primeros pacientes fueron referidos hace años, tanto en el trastorno obsesivo-compulsivo como en pacientes con Gilles de la Tourette ${ }^{84,110}$. En esta revisión analizamos las diferentes dianas quirúrgicas que están apareciendo para tratar pacientes con enfermedades psiquiátricas, así como la disfunción de los circuitos córtico-estriato-tálamo-cortical que conllevan las alteraciones del comportamiento y de las emociones en los trastornos obsesivo-compulsivos, síndrome de Tourette y la depresión refractaria. También revisaremos los signos no motores relacionados con la EP y tratados con estimulación del NST.

\section{Anatomía del circuito límbico}

Es bien conocido, basado en el modelo clásico de funcionamiento de los GB que toda la información cortical es procesada a través de circuitos segregados del estriado que proyecta sobre el globo pálido hacia el tálamo y nuevamente a la corteza ${ }^{5,27,89}$. Esto ha dado soporte anatómico a que existan subdivisiones dentro de los GB como regiones motoras, asociativas y límbicas. Las proyecciones sensitivo-motoras procesarían la información motora, los territorios asociativos la información cognitiva y los límbicos la información emocional dentro de los GB.

Existe evidencia de que la disfunción en el funcionamiento de los GB se muestra por un comportamiento estereotipado con movimientos repetitivos dentro de trastornos del comportamiento, o bien con la aparición de movimientos anormales (disquinesias). Esto se ha podido comprobar en animales de experimentación en los cuales el bloqueo de diferentes porciones del GPe (globo pálido externo) o del estriado dorsal (putamen) con bicuculina (antagonista GABAégico) en monos normales, conlleva la aparición de corea o movimientos mioclónicos si las inyecciones son realizadas en las porciones motoras de dichas estructuras $^{35,124}$. Mientras que comportamientos de hiperactividad, déficit de atención con estereotipias o alteraciones en acciones volitivas se apreciarían cuando las inyecciones están realizadas en las regiones límbicas del estriado, del GPe o NST (en esta estructura con el agonista GABAérgico muscimol) $)^{35,61,124}$. Como conclusión, podemos decir que la actuación sobre diferentes proyecciones anatómicas funcionalmente distintas de los GB, induce diferentes comportamientos en primates. 
FIGURA 1

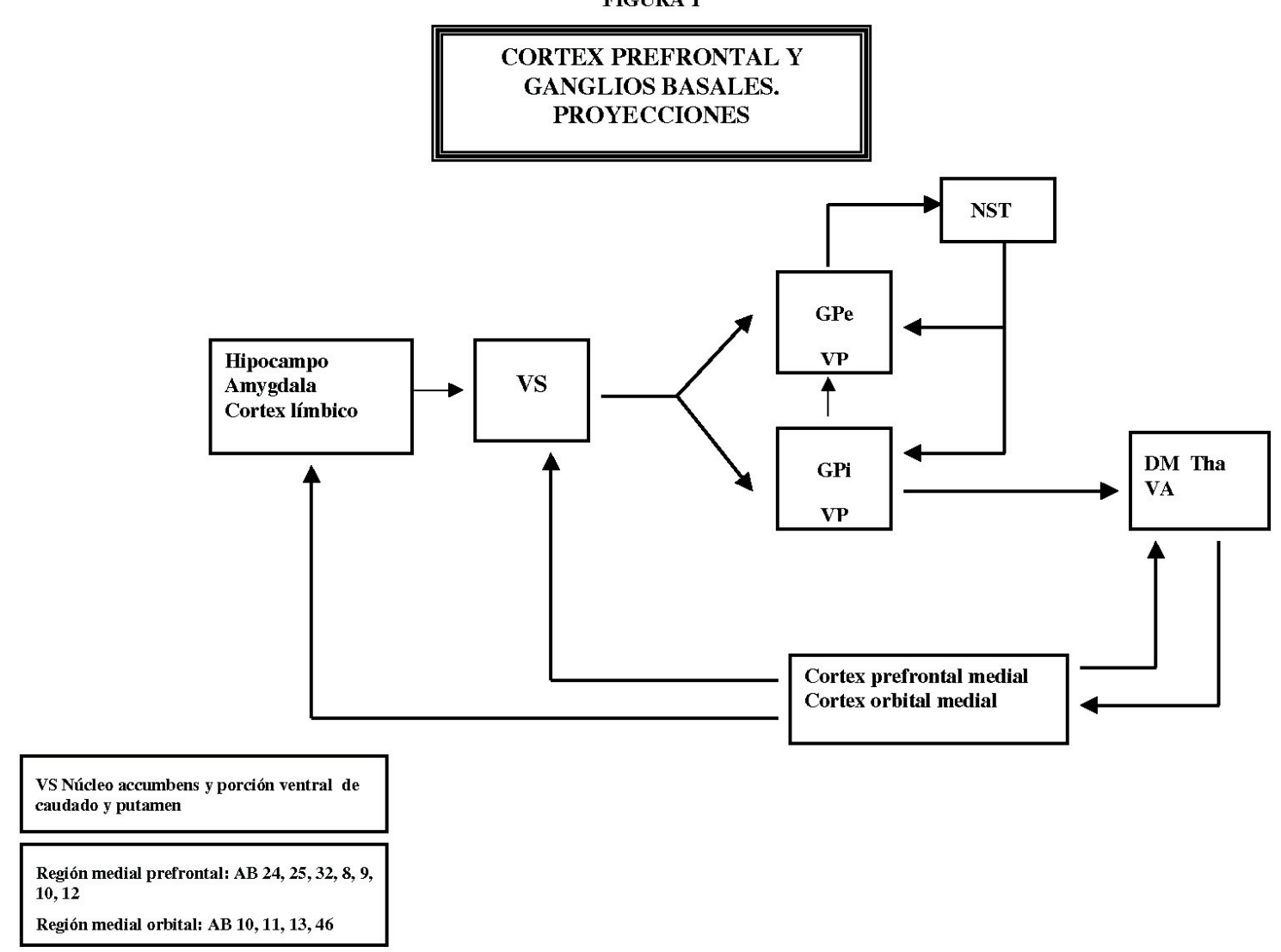

Figura 1. Esquema de las proyecciones desde el córtex prefrontal a ganglios basales. AB: areas de Bordmann. DM Tha: dorsomedial del tálamo. GPi: globo pálido interno. GPe: globo pálido externo. NST: núcleo subtalámico. VA: núcleo anterior del tálamo. VP: pálido ventral. VS: estriado ventral.

Toda la información emocional o afectiva es transmitida a través de las porciones límbicas de los GB. Así el hipocampo, amígdala, áreas límbicas y paralímbicas envían sus proyecciones al estriado ventral (VS: ventral striatum $)^{5,89}$. Esta estructura, el VS, es capital en las emociones y mecanismos de recompensa y está básicamente formada por la porción ventral del caudado y putamen y por el núcleo accumbens (NAcc) $)^{47,48,80}$. Desde el VS, se envían proyecciones sobre el pálido ventral (VP), que es considerado como la principal eferencia desde los GB sobre el tálamo proyectando sobre el núcleo dorso medial (DM), ventral anterior y núcleos intralaminares 5 . El núcleo DM y los diferentes núcleos talámicos, cerrarían el circuito proyectando sobre el córtex cingular anterior (CCA) (área 24 de Brodmann) y córtex órbito frontal (COF) (áreas $10,11,13,43$ de Brodmann) ${ }^{38}$. Las proyecciones tálamocorticales pasan por el brazo anterior de la cápsula interna (CI) en su porción ventral y es la diana de la capsulotomía ${ }^{57}$. (Figura 1).

El córtex prefrontal a su vez proyecta sobre todas las estructuras anatómicas de las cuales recibe aferencias, así envía proyecciones sobre el DM, núcleos intralaminares talámicos y sobre los GB como núcleo caudado, putamen, GP y el núcleo Accumbens (Nacc) $)^{31,38,40}$. El córtex medial prefrontal (áreas $8,9,10,24,25$ y 32 de Brodmann) y el COF (áreas 10,11,13) proyectan sobre el VS, amígdala y córtex temporal medial y esto constituye el sustrato anatómico del comportamiento emocional, instintivo y de recompensa ${ }^{38,47,48}$. Mientras que el córtex prefrontal lateral, conectaría con las porciones laterales del caudado y núcleos laterales talámicos siendo estructuras anatómicas relacionadas con el sustrato cognitivo, ejecutivo y del comportamiento. El VS no recibe aferencias motoras, premotoras ni de área motora suplementaria (AMS) $)^{31,38,47,48}$. Por lo tanto, el VS y el NAcc son estructuras anatómicas directamente relacionadas con el córtex prefrontal y por tanto con las emociones y sus comportamientos, así como con sus procesos patológicos como puede ocurrir en los trastornos obsesivo-compulsivos (TOC), síndrome de Tourette (ST) y depresión ${ }^{4}$. Así, todos ellos tienen en común que presentan una anomalía a nivel corticoestriado-tálamo-cortical. 


\section{Cirugía de la EP. Aspectos motores y no motores}

Con los conocimientos actuales, la EP es considerada como una enfermedad neuro-psiquiátrica y no únicamente como un problema motor ${ }^{3}$. Los distintos tratamientos dopaminérgicos, así como la cirugía en las diferentes dianas (GPi y NST) revierten la acinesia, el temblor y rigidez. La estimulación del NST (DBS-NST) realizada en la región sensitivo-motora del núcleo mejora significativamente los signos capitales de la enfermedad, los signos axiales y las fluctuaciones motoras, como son las disquinesias y bloqueos motores ${ }^{50,64,87,94,98,114}$. La estimulación induciría una inhibición funcional similar a la lesión, aunque su mecanismo de acción no es del todo conocido. Sin embargo, al ser en NST una estructura pequeña (160 mm cúbicos) $)^{125}$, la estimulación puede alcanzar las regiones límbicas y asociativas del NST, produciendo efectos adversos como manía, risa, gritos, conducta agresiva, depresión y trastornos psiquiátricos ${ }^{15,65,67,95,109,123}$. Todos estos fenómenos pueden llevar a tener que disminuir los parámetros de estimulación o a cambiar los contactos activos en ocasiones, pudiendo conllevar una reducción del beneficio motor de los pacientes intervenidos.

En los últimos años, en las evaluaciones clínicas de los pacientes con EP, se está dando importancia a los aspectos no motores de la misma. Una de las alteraciones del comportamiento que presentan los pacientes es un trastorno en el control de impulsos (ICD) como el juego patológico, compras compulsivas, hipersexualidad, así como otros comportamientos compulsivos, como pueden ser el excesivo hobbismo o excesivo uso de internet (punding $)^{29,72,115,116}$. El ICD es un problema poco estudiado y que actualmente comenzamos a conocer pudiendo encontrase hasta en un $14 \%$ de los pacientes con EP y en un $17 \%$ en los tratados con agonistas dopaminérgicos (DA) ${ }^{29,117,118,120}$.

También en este mismo contexto, se han evaluado pacientes tratados con DBS-STN e ICD. Para algunos autores, el beneficio en el ICD tras la cirugía podría estar relacionado con la reducción de los dopaminérgicos $\mathrm{DA}^{9,62}$. Otros autores, por el contrario, han referido la aparición de ICD en pacientes tratados con DBS a pesar de la reducción de agonistas $\mathrm{DA}^{49,72,104}$. Por último en otros estudios realizados, la estimulación del núcleo subtalámico en pacientes jóvenes, aumentaría el riesgo de desarrollar ICD y en este contexto, no únicamente existiría un incremento en presentar un cuadro de impulsividad, sino también un mayor riesgo de suicidio ${ }^{12,55,98,103,104,119}$.

Recientemente se ha publicado que la estimulación subtalámica puede inducir a comportamientos compulsivos, sobre todo cuando existen condiciones de conflicto $^{36}$. Este comportamiento se ha podido apreciar en pacientes con EP en los cuales los tests realizados mostraron una incapacidad para modular sus respuestas al comparar un grupo tratado quirúrgicamente (n: 17) versus pacientes con medicación DA (n:15) ${ }^{36}$. Por tanto, podemos concluir que la estimulación del NST puede conllevar comportamientos impulsivos en algunos pacientes, similares a los de ICD en los pacientes con EP tratados con agonistas DA. Fisiopatológicamente, se trataría de dos mecanismos diferentes en la impulsividad, uno de ellos relacionado con la medicación dopaminérgica, que deriva de los niveles elevados de DA en los receptores D2 estriatales y un segundo mecanismo, sería la estimulación de la porción límbica del NST, en el cual incluso existe una reducción de DA, pero al ser inhibida la actividad del NST, los pacientes mostrarían una impulsividad por la incapacidad para modular decisiones en situaciones de conflicto ${ }^{36}$. Ambas situaciones manifestarían un cuadro clínico similar.

Estos hallazgos en pacientes tratados quirúrgicamente en el NST, estarían en relación con estudios realizados previamente en dicha estructura anatómica en ratas, induciendo una respuesta anormal en la toma de decisiones tras lesionar el NST ${ }^{13,14}$. Los experimentos mostraron que en animales parkinsonizados tras inyectarles 6-OHDA (hidroxidopamina), la lesión del núcleo producía un gran beneficio motor pero aumentaba la respuesta anticipatoria, la conducta perseverativa así como el déficit de atención ${ }^{14}$. En un estudio ulterior, se pudo apreciar que si bien decrecía el tiempo de reacción tras la lesión del NST, existía un incremento en las respuestas prematuras llevando a un déficit de comportamiento. Por tanto parece que el NST puede tener un papel en los GB de selección y de inhibición y que la respuesta anticipatoria conllevaría un mayor número de errores en los animales, pudiendo ser definida como una "disquinesia del comportamiento" ${ }^{36}$. El mismo comportamiento se ha descrito tras estimulación del NST en los animales intervenidos ${ }^{25}$. La conclusión de dichos estudios, demuestra que la manipulación, induciendo una inhibición del NST, puede conllevar comportamientos impulsivos $^{36,108}$.

Todos estos estudios anatómicos y clínicos, nos llevan al concepto de que el NST es un potente regulador de los GB en funciones tanto motoras (su lesión o inhibición induce hemicorea/balismo), cognitivas como emocionales (su lesión o inhibición produce conducta impulsiva y anticipatoria) y nos sugiere que juega un importante papel central como modulador en el circuito cortico-estriatotálamo-cortical que media los circuitos funcionalmente diferentes como son el motor y el límbico ${ }^{5,27,88}$. El NST por un lado recibe la vía hiperdirecta desde el córtex (COF y córtex premotor, AMS y córtex motor primario) ${ }^{81}$ y por otro recibe aferencias desde el estriado a través del GPe, con la misma información aunque diferentemente procesada, por lo cual modula la eferencia asociativa y límbica hacia el GPi y $\mathrm{SNr}$ (sustancia negra reticulata), los núcleos de salida de los GB hacia el tálamo ${ }^{81}$ (Figura 1). 


\section{Estimulación cerebral en los trastornos obsesivo- compulsivos}

El trastorno obsesivo-compulsivo (TOC) se caracteriza por la aparición de ideas obsesivas y recurrentes (obsesiones) unidas a actos motores repetitivos (compulsiones) que llevan al paciente a provocar pérdidas de tiempo significativas y un bloqueo de cualquier actividad, llegando en ocasiones a incapacitarlo totalmente ${ }^{106}$. La incidencia de esta patología en la población es del $2 \%$, de los cuales un $30-40 \%$ de los pacientes serían refractarios a los distintos tratamientos convencionales ${ }^{88}$.

Los estudios funcionales de neuroimagen en el TOC muestran que la medición del flujo sanguíneo cerebral regional (rCBF) de los pacientes en PET (tomografía por emisión de positrones), al ser comparada con sujetos normales, ha mostrado una hiperactividad en el COF, CCA y estriado ${ }^{32,91,92}$. Dicha actividad estaría directamente relacionado con el cuadro clínico, ya que se incrementa durante la provocación de los síntomas de los pacientes y se reduciría con el tratamiento médico ${ }^{92}$. La hiperactividad del COF, estaría relacionada con la gravedad del TOC, de esta manera los pacientes con una buena respuesta clínica tras la cirugía (DBS en VS/VC) muestran una modulación de la hiperactividad del COF, área subgenual CCA, estriado, GP y tálamo. Esto se ha encontrado en 6 pacientes con TOC tratados quirúrgicamente, mientras que dicha hiperactividad se mantenía en los pacientes considerados como refractarios ${ }^{92}$.

Nuttin fue el primer neurocirujano que colocó un sistema de estimulación en cuatro pacientes con TOC refractarios a tratamiento médicos, implantando los electrodos en la misma diana donde se realizaba las capsulotomías en 1999 (brazo anterior de cápsula interna en su porción más ventral: CI ${ }^{84}$. Como hemos visto previamente, por esta zona pasan las proyecciones desde en $\mathrm{CM} / \mathrm{Pf}$ y núcleos intralaminares del tálamo hacia el COF y áreas prefrontales $^{57,84,85}$. Desde entonces, distintos autores han trabajado en esta diana con buenos resultados, ya que se han publicado reducción significativas en las escalas Y-BOCS (Yale Brown Obsessive Compulsive Scale), que es la que mejor evalúa estos pacientes y se han descrito en el seguimiento, un significativo número de pacientes con criterios de remisión (Tabla 1) 1,40,42,56,85,107. También los pacientes con TOC con esta diana quirúrgica mejoran tras la intervención la depresión, evaluados con la escala de Hamilton y la ansiedad, que son problemas coadyuvantes en esta patología. Sin embargo, la diana exacta es variable según los diferentes autores, así unos colocan el electrodo en la cápsula interna $(\mathrm{CI})^{1,7,84,85}$ y otros en $\mathrm{VS} / \mathrm{CI}$, o bien Nacc/CI, aunque esta estructura formaría parte del VS $8,37,41,42,43,44,56,107$. Así, en algunos estudios clínicos, colocan los contactos de estimulación dorsales (contactos 2 y 3 ) en la CI, mientras los más ventrales (contactos 0 y 1 ) en el VS o bien Nacc, sin llegar a especificar los contactos clínicamente activos en las diferentes publicaciones ${ }^{8,43,107}$.

En un estudio de revisión de cuatro centros con experiencia en DBS para TOC en dicha diana quirúrgica, VS-Nacc, se ha descrito el beneficio progresivo de los pacientes, ya que la gravedad de la enfermedad en las escalas de evaluación fue reduciéndose en las sucesivas revisiones ${ }^{44}$. Así, a los tres meses tras la cirugía, la Y-BOCS disminuyó una media de 12.5 puntos en la escala (34.0 basal a 21.0 postimplante) y el porcentaje de pacientes que tenían criterios de "respondedores" (reducción de un $35 \%$ en la escala o mayor) se incrementó de un $28 \%$ al mes de la cirugía a un $61.5 \%$ en la última revisión ${ }^{44}$. Por otro lado, el cuadro de depresión y de ansiedad de estos pacientes también mejora durante el seguimiento de los mismos, existiendo a los 36 meses del implante una reducción del $43.2 \%$ en la escala de Hamilton para la depresión y de un $58.7 \%$ en la de HARS para la ansiedad. En el mismo trabajo los autores describen que la diana quirúrgica ha ido acercándose a la comisura anterior (CA), así en los primeros pacientes intervenidos estaba a $15 \mathrm{~mm}$ anterior a la misma y los pacientes intervenidos posteriormente en el tiempo, la diana estaba próxima a la CA y más ventral, estimulando VS y NAcc ${ }^{44}$.

Por último, recientemente el grupo de la universidad de Colonia ha publicado un estudio doble ciego con estimulación del NAcc derecho en 10 pacientes $^{56}$. Tras la intervención y estabilización de los mismos, la evaluación se realizó con la estimulación "on" o bien "off" durante un periodo de tres meses con parámetros de estimulación fijos. El estudio ha mostrado una reducción de las escalas de una basal de 32.2 a 27.9 en situación "on" estimulación (p: 0.033) y "off" estimulación de 31.1 en la escala de YBOCS. Según los autores, tras 12 meses de estimulación del NAcc derecho, sólo uno de los 10 pacientes se consideró como respuesta total (reducción de la Y-BOCS del $35 \%$ o superior) tras un año de estimulación cuatro pacientes alcanzaron una respuesta parcial (mejoría entre 25-35\% con respecto a la basal) ${ }^{56}$. Actualmente los autores, durante el segundo año de estimulación, están estudiando la variación en los parámetros de estimulación con una cirugía unilateral en cada paciente y su respuesta clínica.

Por otro lado, otros autores encontraron que pacientes con EP que también tenían TOC y que fueron intervenidos con DBS-NST, tras la cirugía mejoraron no sólo los aspectos motores del Parkinson sino también la Y-BOCS ${ }^{34,74}$. Esto llevó a un estudio aleatorio, doble ciego con la diana en la parte límbica del NST realizado por diferentes centros en Francia. Tras la cirugía y estabilización de la estimulación, se eligieron al azar 16/18 pacientes, tratándose en dos fases de 3 meses cada una, para hacer estimulaciones "on" u "off", separadas por un mes de estimulación simulada ${ }^{75}$. Se comprobó que con la estimulación del NST, la Y-BOCS era 
Tabla 1

Estudios de DBS para TOC

\begin{tabular}{|c|c|c|c|c|c|}
\hline Autor & $\begin{array}{c}\mathbf{N}^{\circ} \\
\text { pacientes }\end{array}$ & $\begin{array}{l}\text { seguimiento } \\
\text { (meses) }\end{array}$ & Diana quirúrgica & Respuesta clínica & Comentarios \\
\hline $\begin{array}{l}\text { Nuttin y cols. } \\
1999^{84}\end{array}$ & 4 & & CI & $\begin{array}{l}3 / 4 \text { pac algún } \\
\text { beneficio }\end{array}$ & $\begin{array}{l}\text { En un paciente } \\
\text { estudio doble ciego }\end{array}$ \\
\hline $\begin{array}{l}\text { Nuttin y cols. } \\
2003^{85}\end{array}$ & 6 & 21 & $\begin{array}{l}\text { CI } \\
Y: 3.5 \text { anterior a CA } \\
\mathrm{X}: 13-14 \\
\mathrm{Z}: 0\end{array}$ & $\begin{array}{l}\text { Y-BOCS } \\
32.3-19.8 \text { (off -on } \\
\text { stimulatiom) }\end{array}$ & $\begin{array}{l}\text { - Contacto distal ( } 0 \text { ) en NAcc } \\
\text { - Doble ciego ( } 3 \text { meses) }\end{array}$ \\
\hline $\begin{array}{l}\text { Abelson y } \\
\text { cols. } 2005^{1}\end{array}$ & 4 & 23 & CI & $\begin{array}{l}\text { Gran beneficio en } \\
\text { un paciente }(73 \%) \\
\text { y moderado } \\
\text { en otro }\end{array}$ & $\begin{array}{l}\text { - Contacto distal }(0) \text { en la base de } \\
\text { la CI en relación con NAcc } \\
\text { - Evaluación on-off ( } 3 \text { semanas) } \\
\text { - Un paciente suicidio }\end{array}$ \\
\hline $\begin{array}{l}\text { Sturm y cols. } \\
2003^{107}\end{array}$ & 4 & $24-30$ & $\begin{array}{l}\text { Nacc/CI } \\
\text { Y: } 2.5 \text { anterior AC } \\
\text { X: } 6.5 \\
\text { Z: } 4.5\end{array}$ & $\begin{array}{l}\text { Reducción signifi- } \\
\text { cativa en } 3 / 4 \\
\text { (no describe escalas) }\end{array}$ & $\begin{array}{l}\text { - } 2 \text { contactos en NAcc y } 2 \text { en CI } \\
\text { - Unilateral derecho }\end{array}$ \\
\hline $\begin{array}{l}\text { Anderson y } \\
\text { Ahmed }^{7}\end{array}$ & 1 & 3 & $\begin{array}{l}\text { CI } \\
\text { X: } 18 \\
\text { Y: } 3 \text { anterior DC } \\
\text { Z: ? }\end{array}$ & Y-BOCS: $34-7$ & 2 voltios estimulación \\
\hline $\begin{array}{l}\text { Greenberg y } \\
\text { cols. } 2006^{43}\end{array}$ & 8 & 36 & $\begin{array}{l}\text { CI/NAcc } \\
\text { Inmediatamente } \\
\text { rostral a CA }\end{array}$ & $\begin{array}{l}4 / 8>35 \% \text { reduc- } \\
\text { ción Y-BOCS } \\
\text { (Y-BOCS: } 34-22 \\
(\mathrm{p}<0.001)\end{array}$ & $\begin{array}{l}\text { - Contactos distales: VS y NAcc } \\
\text { - Contactos dorsales: CI } \\
\text { - Mejoría depresión y ansiedad } \\
\text { - Hemorragia asintomática }\end{array}$ \\
\hline $\begin{array}{l}\text { Aouizerate y } \\
\text { cols } 2004^{8}\end{array}$ & 1 & 15 & $\begin{array}{l}\text { CI/NAcc/Caudado } \\
\mathrm{X}: 8.9 \\
\mathrm{Y}: 36.5 \text { anterior a AC } \\
\mathrm{Z}:-3\end{array}$ & $\begin{array}{l}\text { Remisión OCD } \\
\text { (Y-BOCS < 16) }\end{array}$ & $\begin{array}{l}\text { - Contactos distales: NAcc } \\
\text { - Contactos dorsales: N.Caudado/CI } \\
\text { - Mejoría depresión y ansiedad }\end{array}$ \\
\hline $\begin{array}{l}\text { Mallet y cols } \\
2002^{74}\end{array}$ & 2 & 6 & $\begin{array}{l}\text { NST } \\
\text { (porción límbica) }\end{array}$ & $\begin{array}{l}58 \% \text { y } 64 \% \text { mejoría } \\
\text { de la Y-BOCS }\end{array}$ & Mejoría de Parkinson \\
\hline $\begin{array}{l}\text { Fontaine y cols } \\
2004^{34}\end{array}$ & 1 & 12 & $\begin{array}{l}\text { NST } \\
\text { X: } 12.5 \text { y } 10 \\
\text { Y: } 0.5 \text { y } 3.8 \text { post. a MCP } \\
\text { Z: }-1.5 \text { y }-4.8\end{array}$ & $\begin{array}{l}\text { Remisión OCD } \\
\text { 32-1 (Y-BOCS) }\end{array}$ & Mejoría de Parkinson \\
\hline $\begin{array}{l}\text { Mallet y cols } \\
\qquad 2008^{75}\end{array}$ & $16 / 18$ & 3 & $\begin{array}{l}\text { NST } \\
2 \mathrm{~mm} \text { anterior } \\
1 \mathrm{~mm} \text { medial a diana } \\
\text { de Parkinson }\end{array}$ & $\begin{array}{l}\text { Reducción Y-BOCS } \\
28-19 \text { (sham-on) } \\
\mathrm{p}=0.01\end{array}$ & $\begin{array}{l}\text { - Estudio doble ciego con sham } \\
\text { - } 1 \text { hemorragia intracerebral } \\
\text { - } 2 \text { infecciones del sistema } \\
\text { - Trastornos cognitivos: } 3 \text { hipomanías } \\
2 \text { ansiedad }\end{array}$ \\
\hline $\begin{array}{l}\text { Jiménez-Ponce } \\
\text { y cols } 2009^{60}\end{array}$ & 5 & 12 & $\begin{array}{l}\text { Pedúnculo talámico inferior } \\
\text { X: } 3.5 \\
\text { Y: } 5 \text { post AC } \\
\text { Z: } 0\end{array}$ & $\begin{array}{l}\text { Y-BOCS }(35-17.8) \\
(p<0.001\end{array}$ & $\begin{array}{l}\text { Contactos implantados cerca del fórmix } \\
\text { - } 3 \text { pacientes continuaron con } \\
\text { consumo de drogas }\end{array}$ \\
\hline $\begin{array}{l}\text { Franzini y cols. } \\
\qquad 2009^{41}\end{array}$ & 2 & $24-27$ & $\begin{array}{l}\text { Nace } \\
\text { X } 16 \text { ant. a MCP } \\
\text { X: } 3 \text { lateral a IC } \\
\text { Z: }-2\end{array}$ & Y-BOCS: $38-22$ y $30-20$ & \\
\hline $\begin{array}{l}\text { Goodman y cols. } \\
2010^{37}\end{array}$ & 6 & 12 & $\mathrm{VS} / \mathrm{CI}$ & $\begin{array}{l}4 / 6 \text { mejoría del } \\
35 \% \text { Y-BOCS }\end{array}$ & $\begin{array}{l}\text { Pacientes no mejoraron durante } \\
\text { el periodo de simulación }\end{array}$ \\
\hline $\begin{array}{l}\text { Huff y cols } \\
2010-05-28^{56}\end{array}$ & 6 & 12 & $\mathrm{NaCC}$ & $\begin{array}{l}1 \text { paciente Y-BOCS }>35 \% \\
4 \text { pacientes Y-BOCS: } 25-20 \% \\
\text { Reducción global: } p: 0,03\end{array}$ & $\begin{array}{l}\text { Estimulación NAcc derecho } \\
\text { Doble ciego estimulación }\end{array}$ \\
\hline
\end{tabular}


significativamente inferior a la estimulación simulada (28 vs 19). La diana quirúrgica se situó $1 \mathrm{~mm}$ medial y $2 \mathrm{~mm}$ anterior a la diana para la EP en dicha estructura. El estudio mostró 15 efectos adversos serios incluyendo una hemorragia cerebral y 2 infecciones. Entre las complicaciones de esta diana quirúrgica, la estimulación en algunos pacientes indujo impulsividad e hipomanía similar a los pacientes tratados en la misma diana para $\mathrm{EP}^{75}$.

Actualmente, una paradoja en este campo es cómo la estimulación del NST en pacientes con EP, puede inducir impulsividad y desinhibición en sus comportamientos, mientras que la misma estructura puede mejorar las obsesiones y compulsiones en los pacientes con TOC. Esto podría indicar que ambos fenómenos, impulsividad y desinhibición por un lado y obsesiones y compulsiones por otro, pudieran ser opuestos dentro del campo del comportamiento y las emociones.

Los parámetros de estimulación, en las distintas publicaciones, son muy variables, entre 4-10 voltios, según los distintos autores $1,8,37,41,43,60,85,107$. Así Goodman y cols estimularon con 8 voltios y Abelson con 10 voltios en CI, por lo que se debe asumir que el campo de estimulación es grande y puede alcanzar diferentes estructuras anatómicas además de la deseada ${ }^{1,41}$. Anderson y Ahmed son los únicos autores que refieren estimulación con 2 Vols a nivel de CI con buen resultado clínico ${ }^{7}$. Los autores cuya diana fue el NST refieren estimulaciones alrededor de 3 Vols similar a los pacientes con $\mathrm{EP}^{34,73,74}$. Los electrodos también han variado entre los electrodos modelo 3387 y 3389 (10.5 y $7.5 \mathrm{~mm}$ de estimulación activa; Medtronic) para estimulación del Nacc/VS/CI o NST. Franzini es el único autor que empleó el 3389 para Nacc, mientras que todos los autores que fueron al NST emplearon el electrodo $3389^{34,37,74}$ (Tabla 1).

La experiencia de los trabajos reportados en este campo nos lleva a concluir que existen diferentes dianas quirúrgicas que pueden llegar a modular la actividad patológica de los circuitos órbito-frontal y córtex medial prefrontal a través de una disrupción o modulación de sus fibras entre córtex frontal y tálamo o bien dentro del propio VS. Ambas dianas, VS/CI y NST, actuarían a distintos niveles con resultados clínicos similares. También se ha observado en las revisiones evolutivas, que los pacientes intervenidos mantienen una mejoría progresiva, lo que indicaría que la modulación de los circuitos no es inmediata, como ocurre en los trastornos del movimiento, sino que lleva más tiempo en alcanzar el beneficio terapéutico considerado como óptimo.

\section{Síndrome de Gilles de la Tourette}

El síndrome de Gilles de la Tourette (SGT) es una alteración neuropsiquiátrica que consiste en la aparición de tics motores tanto faciales, fónicos como vocales desde

\section{Post-synaptic targets for striatal afferent systems}

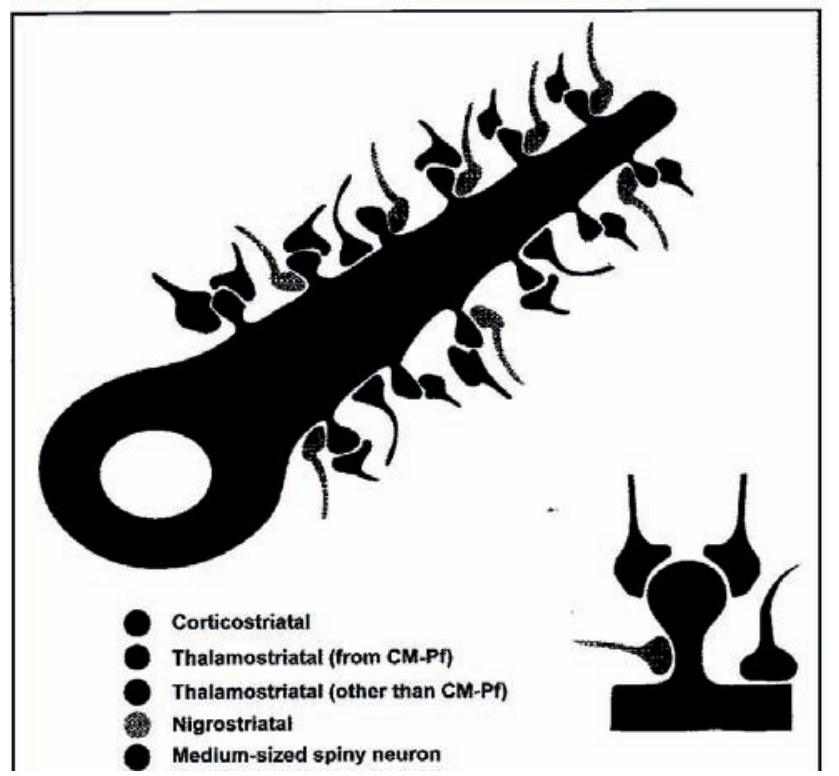

Figura 2. Las neuronas espinosas del estriado (spiny neuron) reciben aferencias de la corteza cerebral (ácido glutámico como neurotransmisor), del núcleo centro mediano parafascicular (CM/Pf) del tálamo (ácido glutámico como neurotransmisor) y de otros núcleos talámicos como el ventral anterior y núcleos intralaminares (ácido glutámico como neurotransmisor). Además recibe aferencias dopaminérgicas desde la SNc (sustancia nigra pars compacta). La dopamina solo es liberada cuando va a realizarse un movimiento por parte del sujeto. Existe entre las aferencias glutamatérgicas y las dopaminérgicas un equilibrio que en caso de romperse puede generar una patología. Así si la dopamina no es capaz de controlar o inhibir la llegada de aferencias corticales puede inducir la aparición de fragmentos de movimiento similares a las disquinesias.

la niñez. El 96\% de los pacientes con el síndrome lo manifiestan antes de los 11 años de edad ${ }^{58}$. La incidencia de este problema es entre 3-5 casos cada 10.000 habitantes y se trata de una enfermedad autosómica dominante con una penetrancia y fenotipo variables. El cuadro clínico del SGT va evolucionando a lo largo de los años con un incremento de las disquinesias en otras localizaciones como cabeza y extremidades superiores, mientras que en otros pacientes los tics suelen remitir. Por otro lado y en un alto porcentaje de pacientes, existe un cuadro neuropsiquiátrico como déficit de atención, hiperactividad y TOC. Este síndrome conocido tanto por psiquiatras como por neurólogos tiene un sustrato funcional en los $\mathrm{GB}^{4,58}$.

No existen actualmente modelos animales de Tourette que puedan ser estudiados en el laboratorio. Los estudios 
con RM funcional en pacientes, han mostrado un incremento de la actividad a nivel prefrontal, parietal, temporal y cingular anterior ${ }^{90}$. Aunque la fisiopatología del SGT no se conoce con exactitud, puede tratarse de un problema de los GB a nivel estriatal y de la función dopaminérgica. La actividad de descarga tónica desde esta estructura hacia el GPi estaría disminuida, permitiendo movimientos o tics incontrolados hacia el tálamo y la corteza, similar al modelo de hipercinesia. Dicha actividad anormal estaría en las neuronas estriatales (neuronas espinosas) que responderían patológicamente descargando al estímulo dopaminérgico e induciendo los tics, sin poder inhibir los movimientos (disquinesias) creando una especie de focos aberrantes de descarga sin que actualmente se conozca la fisiopatología ${ }^{4}$ (Figura 2). La anomalía del estriado, al estar segregado funcionalmente, hace que el cortejo de tics pueda ser variable; bien simples, complejos o compulsiones dependiendo del lugar en que se genere el foco en el estriado.

Así en los tics simples o vocales, existiría una actividad anormal a nivel motor, premotor y a nivel cingular y del córtex órbito frontal para los tics motores complejos como las compulsiones ${ }^{4}$. Por tanto el SGT se trataría de una desinhibición del circuito cortico-estriado-tálamocortical con un origen a nivel estriatal. Esta actividad aberrante mejora o queda suprimida por los antagonistas dopaminérgicos y por los inhibidores selectivos de la recaptación de serotonina ${ }^{4}$.

Los tics además de motores y fónicos, pueden ser también obsesiones motoras como compulsiones. Las palabras obscenas, coprolalia o ecolalia, son consideradas como un cuadro obsesivo, desde el punto psiquiátrico, que acompaña a los tics motores dentro del SGT. Debemos de considerar los tics y las obsesiones como disquinesias ya que son fragmentos liberados, motores o cognitivos desde el estriado sobre el tálamo ${ }^{93}$. La única diferencia entre los tics y las obsesiones sería el origen en diferente región dentro del estriado. En este contexto, el GPi puede ser una buena diana quirúrgica para tratar cualquiera de estas disquinesias, tanto a nivel posteroventral, región sensitivomotora, como la anteromedial o región límbica del GPi.

Cuando los tratamientos conservadores fracasan en los pacientes afectos de SGT grave, la estimulación cerebral puede constituir una alternativa terapéutica válida. Estos pacientes refractarios a la terapia médica deben ser valorados por neurólogos y psiquiatras como candidatos a la cirugía. Para ello, están publicados unos criterios de inclusión/exclusión tanto médicos como quirúrgicos para intervenir esta patología ${ }^{86}$. La evaluación clínica de los pacientes se realiza con la Yale Global Tic Severity Scale (YGTSS), ya que la escala evalúa los tics motores, los fónicos y la afectación de los mismos en la vida del paciente, así como la gravedad global de los mismos. En
1999, Vanderwalle y cols introdujeron la estimulación cerebral en tres pacientes que sufrían esta patología, con buena respuesta clínica inicial. La diana quirúrgica fue el tálamo, similar a la diana de las lesiones realizadas por Hassler y Dieckmann en 1970. Los núcleos elegidos, según el artículo original fueron el ventral oral interno (Voi) y $\mathrm{CM} / \mathrm{Pf}^{53,110}$.

Diferentes dianas han sido probadas en el SGT desde entonces y unos 60 pacientes han podido ser intervenidos y publicados ${ }^{11,28,33,66,73,83,100-102,111,113}$ (Tabla 2). El GPi, tanto en su porción motora como la anteromedial o límbica y el tálamo (CM/Pf y Voi) han sido las más frecuentemente utilizadas. La elección del GPi se debe a considerar los tics como disquinesias y por tanto un problema exclusivo motor, mientras que en los pacientes con una afectación psiquiátrica hay autores que prefieren elegir el tálamo como diana quirúrgica. En ambas dianas, los resultados clínicos han podido ser comparables (66\% de mejoría en la YGTSS) pero el número de pacientes intervenidos es bajo como para sacar conclusiones sobre cualquiera de ellas. Los resultados sobre las alteraciones del comportamiento de los pacientes son variables y actualmente no se conoce con exactitud. En los pacientes en los cuales el cuadro psiquiátrico es el dominante y el motor es menor se ha probado la estimulación en Nacc/CI similar a los pacientes con TOC, pero son pocos casos y con resultados dispares ${ }^{22,33,66}$.

Otros pacientes han sido intervenidos con GPi y CM/ Pf a la vez, aunque no está demostrado en las escalas de evaluación clínica, que la suma de las dos dianas aporte un beneficio nuevo a los pacientes ${ }^{2,39}$. Por tanto, en esta enfermedad la diana quirúrgica no está bien definida y la estimulación en los mismos circuitos, pero en diferentes puntos, puede producir resultados similares.

\section{Estimulación profunda en la depresión}

La depresión es una de las enfermedades más prevalentes actualmente en nuestra sociedad ya que afecta a un $4 \%$ de la población general y un $15 \%$ de la misma ha tenido depresión alguna vez en su vida ${ }^{6}$. De los pacientes afectos de una depresión mayor o grave, a pesar de los diferentes tratamientos médicos, psicoterápicos o bien terapia electroconvulsiva (TEC), un $20 \%$ de los mismos son considerados como refractarios a los mismos ${ }^{6,30}$. Los pacientes con depresión resistentes eran intervenidos, hasta hace unos años, con una cirugía estereotáctica ablativa como la capsulotomía, tractotomía subcaudada o bien cingulotomía. Actualmente se ha iniciado la estimulación cerebral en esta enfermedad.

La imagen funcional en esta patología, ha mostrado que los pacientes con tristeza o bien con depresión, presentan una hiperactividad en la región subgenual (área 25 de Brodmann) y que la misma se revierte en los pacientes tra- 
Tabla 2

Estudios de DBS para Tourette

\begin{tabular}{|c|c|c|c|c|c|}
\hline Autor & $\begin{array}{c}\mathbf{N}^{0} \\
\text { pacientes }\end{array}$ & $\begin{array}{l}\text { Seguimiento } \\
\text { (meses) }\end{array}$ & $\begin{array}{c}\text { Diana } \\
\text { quirúrgica }\end{array}$ & Respuesta clínica & Comentarios \\
\hline $\begin{array}{l}\text { Visser- } \\
\text { Vanderwalie } \\
\text { y cols. } 2003^{113}\end{array}$ & $\begin{array}{c}3 \\
(42,28,45)\end{array}$ & $\begin{array}{l}\text { CM/Pf, Voi: } \\
\text { X: } 3 \text { y } 5 \\
\text { Y: } 4 \text { anterior MCP } \\
\text { Z. } 0\end{array}$ & $60,12,8$ & Gran mejoria motora y vocal & Cambio en comportamiento sexual \\
\hline $\begin{array}{l}\text { Diederich y } \\
\text { cols. } 2005^{28}\end{array}$ & $\begin{array}{c}1 \\
(27)\end{array}$ & $\begin{array}{l}\text { GPi } \\
\text { X: } 17 \\
\text { Y: } 4 \text { anterior MCP } \\
\text { Z: }-5\end{array}$ & 14 & $\begin{array}{l}\text { Reducción } 73 \% \text { tics vocales } \\
\text { Reducción Y-GTSS }\end{array}$ & \\
\hline $\begin{array}{l}\text { Houeto ycols. } \\
2005^{54}\end{array}$ & $\begin{array}{c}1 \\
(36)\end{array}$ & CM/Pf y GPi & 6 & $\begin{array}{l}\text { Reducción } 70 \% \\
\text { severidad de tics }\end{array}$ & $\begin{array}{l}\text { Problemas psiquiátricos preoperatorios } \\
\text { - GPi implantación zona límbica }\end{array}$ \\
\hline $\begin{array}{l}\text { Welter y cols. } \\
2008^{121}\end{array}$ & $\begin{array}{c}3 \\
(36,30,30)\end{array}$ & CM/Pf y GPI & $60,27,20$ & $\begin{array}{l}\text { Y-GTSS reducción } \\
65 \%, 96 \%, 74 \%\end{array}$ & $\begin{array}{l}\text { - CM/Pf:reducción } 64 \%, 30 \% \text { y } 40 \% \\
\text { - CM/Pfy GPi: no mejoria mayor } \\
\text { - Tics fónicos: reducción } 80 \%-90 \% \text { y } 67 \%\end{array}$ \\
\hline $\begin{array}{l}\text { Flaherty y } \\
\text { cols. } 2005^{33}\end{array}$ & $\begin{array}{c}1 \\
(37)\end{array}$ & $\begin{array}{l}\text { IC/NAcc } \\
\text { X: } 12 \\
\text { Y: punto medio brazo } \\
\text { anterior de CI } \\
\text { Z: }-7\end{array}$ & 18 & Y-GTSS reducción & $\begin{array}{l}\text { - Reducción } 17 \% \text { tics } \\
\text { - Reducción } 20 \% \text { severidad }\end{array}$ \\
\hline $\begin{array}{l}\text { Shields y cols. } \\
2008^{102}\end{array}$ & $\begin{array}{c}1 \\
(40)\end{array}$ & $\begin{array}{l}\text { CM/Pf, Voi } \\
\mathrm{X}: 5 \\
\mathrm{Y}: 4 \text { posterior MCP }\end{array}$ & 3 & $\begin{array}{l}42 \% \text { reducción tics motores } \\
40 \% \text { reducción tics fónicos } \\
46 \% \text { reducción Y-GTSS }\end{array}$ & $\begin{array}{l}\text { El mismo paciente reintervenido (la } \\
\text { estimulacion inducía apatía } \\
\text { en los contactos ventrales } \\
\text { e hipomanía en los dorsales) } \\
\text { Ruptura de electrodo }\end{array}$ \\
\hline $\begin{array}{l}\text { Ackermans } \\
\text { y cols. }^{2}\end{array}$ & $\begin{array}{l}1(45) \\
1(27)\end{array}$ & $\begin{array}{l}\text { CM/Pf, Voi } \\
\text { GPi }\end{array}$ & $\begin{array}{l}12 \\
12\end{array}$ & $\begin{array}{l}\text { Gran reducción en } \\
\text { ambos pacientes }\end{array}$ & $\begin{array}{l}\mathrm{X}: 5 \\
\mathrm{Y}: 4 \text { posterior MCP } \\
\mathrm{Z}:+2 \mathrm{y}+3.5 \\
\mathrm{X}: 21.5 \\
\mathrm{Y}: 4 \text { anterior } \mathrm{MCP} \\
\mathrm{Z}:-3\end{array}$ \\
\hline $\begin{array}{l}\text { Kuhn y cols. } \\
\quad 2006^{66}\end{array}$ & $\begin{array}{c}1 \\
(26)\end{array}$ & $\begin{array}{l}\text { NAcc/CI } \\
\text { X: } 6.5 \\
\text { Y: } 2.5 \text { anterior a AC } \\
\mathrm{Z}:-4.5\end{array}$ & 30 & $\begin{array}{l}\text { Reducción significativa } \\
\text { Y-GTSS: } 4 \mathrm{I} \% \text { reducción }\end{array}$ & Y-BOCS: reducción 50\% \\
\hline $\begin{array}{l}\text { Shahed y cols. } \\
\quad 2007^{101}\end{array}$ & $\begin{array}{c}1 \\
(16)\end{array}$ & $\begin{array}{l}\text { GPi } \\
\text { X: } 22 \\
\text { X: } 3 \text { anterior a MCP } \\
\text { Z: } 4\end{array}$ & 6 & $\begin{array}{l}\text { Tics motores: reducción } 76 \% \\
\text { Tics fónicos: reduccián } 68 \% \\
\text { Y-GTSS global: } 84 \%\end{array}$ & $\begin{array}{l}\text { Y-BOCS: obsesiones } \\
\text { mejoría } 100 \% \\
\text { Compulsiones: } 29 \%\end{array}$ \\
\hline $\begin{array}{l}\text { Bajwa y cols. } \\
2007^{11}\end{array}$ & $\begin{array}{c}1 \\
(48)\end{array}$ & CM/Pf, Voi & 24 & Y-GTSS: reducción 66\% & $\begin{array}{l}\text { Subjetivo paciente reducción } 95 \% \text {, } \\
\text { Mejoría de TOC }\end{array}$ \\
\hline $\begin{array}{l}\text { Maciunas y cols } \\
2007^{73}\end{array}$ & $\begin{array}{c}5 \\
(18-34)\end{array}$ & $\begin{array}{l}\text { CM/Pf, Voi } \\
\mathrm{X}: 4.3 \\
\mathrm{Y}: 4.5 \text { posterior MCP } \\
\mathrm{Z}: 0\end{array}$ & 3 & $\begin{array}{l}\text { Tics motores: reducción } 53 \% \\
\text { Tics fónicos: reducción } 70 \% \\
\text { Y-GTSS: reducción } 44 \%\end{array}$ & \\
\hline $\begin{array}{l}\text { Servello y cols. } \\
\quad 2008^{100}\end{array}$ & $\begin{array}{c}18 \\
(17-47)\end{array}$ & CM/Pf, Voi & 12 & $\begin{array}{l}\text { Y-GTSS: motor }<0.001 \\
\text { Tics fónicos: }<0.001 \\
\text { Global: }<0,001\end{array}$ & $\begin{array}{l}\text { Evaluados "on/off" y } \\
\text { Estimulación simulada }\end{array}$ \\
\hline $\begin{array}{l}\text { Vilela-Filho } \\
2007^{112}\end{array}$ & 2 & GPe & 23 & Reducción 8 t\% & \\
\hline $\begin{array}{l}\text { Dehning y cols } \\
2008^{26}\end{array}$ & 1 & $\begin{array}{l}\text { Gpi } \\
\mathrm{X}: 20 \\
\mathrm{Y}: 3 \text { anterior a } \mathrm{MCP} \\
\mathrm{Z}:-4\end{array}$ & 12 & $\begin{array}{l}\text { Y-GTSS: reducción } \\
\text { frecuencia e intensidad } \\
\text { de los tics }\end{array}$ & \\
\hline
\end{tabular}




\begin{tabular}{|c|c|c|c|c|c|}
\hline Autor & $\begin{array}{c}\mathbf{N}^{0} \\
\text { pacientes }\end{array}$ & $\begin{array}{l}\text { Seguimiento } \\
\quad \text { (meses) }\end{array}$ & $\begin{array}{c}\text { Diana } \\
\text { quirúrgica }\end{array}$ & Respuesta clínica & Comentarios \\
\hline $\begin{array}{l}\text { Neuner y cols } \\
2009^{83}\end{array}$ & 1 & $\begin{array}{l}\text { NAcc } \\
\mathrm{X}: 2.5 \text { anterior } \mathrm{AC} \\
\mathrm{Y}: 6.5 \\
\mathrm{Z}:-4.5\end{array}$ & 36 & $\begin{array}{l}\text { Y-GTSS: reducción de } \\
100-56 \text { puntos }\end{array}$ & Y-BOCS: reducción de 32-15 puntos \\
\hline $\begin{array}{l}\text { Budick y cols } \\
2010^{22}\end{array}$ & 1 & Naec & 30 & $\begin{array}{l}\text { Y-GTSS: empeoramiento } \\
\text { de los tics }\end{array}$ & TOC adyuvante \\
\hline $\begin{array}{l}\text { Gallagher y } \\
\text { cols. }{ }^{39}\end{array}$ & $\begin{array}{c}1 \\
(26)\end{array}$ & $\begin{array}{l}\text { CM/Pf, Voi } \\
\text { Gpi }\end{array}$ & 7 & $\begin{array}{l}\text { Desaparición tics vocales } \\
\text { y marcada mejoria } \\
\text { en los tics de cuello }\end{array}$ & \\
\hline $\begin{array}{l}\text { Martínez-Torres } \\
\text { y cols. }{ }^{77}\end{array}$ & $\begin{array}{c}1 \\
(38)\end{array}$ & NST & 12 & $\begin{array}{l}\text { Reducción en la frecuencia } \\
\text { de los tics } 97 \% \text { reducción } \\
\text { de compulsiones }\end{array}$ & $\begin{array}{l}\text { Mejoría en la EP } \\
\text { Reducci6n } 56 \% \text { medicación } \\
\text { Reducción } 57 \% \text { UPDRS }\end{array}$ \\
\hline $\begin{array}{l}\text { Vernaeken y cols. } \\
\qquad 2009^{111}\end{array}$ & $\begin{array}{c}1 \\
(22)\end{array}$ & $\begin{array}{l}\text { GPi } \\
\text { CM/Pf } \\
\mathrm{X}: 5.5 \text { y } 5 \\
\mathrm{Y}: 3.7 \text { posterior } \mathrm{MCP} \\
\mathrm{Z}: 1.3\end{array}$ & 12 & $\begin{array}{l}\text { GPi no beneficio clínico } \\
\text { Al año tras cirugia talámica } \\
\text { Y-GTS.S: } 92-59(36 \%)\end{array}$ & $\begin{array}{l}\text { Intervenido en } \mathrm{CM} / \mathrm{PF} \text { tras falta } \\
\text { de beneficio en GPi } \\
\text { Importante beneficio TOC } \\
\text { y déficit de atención }\end{array}$ \\
\hline
\end{tabular}

tados por cualquiera de las terapias antes citadas con buena respuesta clínica. Sin embargo, los pacientes considerados como refractarios a cualquier terapia médica, psicoterapia o TEC, mantienen un incremento de la actividad en la región subgenual ${ }^{78,99}$. La hipótesis sobre si la estimulación cerebral pudiera modular la hiperactividad de esta región anatómica ha sido probada por Lozano y Myberg inicialmente en 6 pacientes, en un estudio abierto, que presentaban criterios de depresión refractaria. La estimulación de dicha región se asoció con una mejoría en 4 de los 6 pacientes intervenidos manteniéndose a los 6 meses de la cirugía ${ }^{79}$. Todos los pacientes que mejoraron clínicamente, tuvieron una reducción en la actividad subgenual en el PET. Los autores sugirieron que la interupción en el circuito límbico a nivel cortical con la estimulación, revertía los síntomas en pacientes refractarios ${ }^{79}$.

El mismo grupo y en una extensión del estudio, publicó un artículo basado en 20 pacientes, con observación clínica a los 6 meses de la cirugía, en el cual se ponía de manifiesto que el $60 \%$ respondían a la estimulación y en el $35 \%$ se observó una remisión de la enfermedad ${ }^{71}$. Los criterios de respuesta eran si los pacientes alcanzaban un beneficio del $50 \%$ o mayor reducción en los 17 items de la escala de Hamilton (Hamilton Rating Scale for depression; HRSD17) y los criterios de remisión eran si la escala llegaba a 7 puntos o inferior. El beneficio postcirugía permaneció estable durante 12 meses $^{71}$. Sin embargo, no se han encontrado diferencias significativas entre los que respondían al tratamiento y los que no respondían, tanto en relación con la colocación de los electrodos como en los estudios de tractografía en la $\mathrm{RM}^{51,71}$.

Varios grupos quirúrgicos han publicado pacientes tratados con estimulación en diferentes dianas quirúrgicas. El trabajo clínico con un mayor número de casos ha sido publicado por Malone y cols con estimulación del VS/CI en 15 pacientes con depresión refractaria ${ }^{76}$. Se consideró que un $40 \%$ de los pacientes intervenidos habían respondido bien al tratamiento, cuando eran estudiados con la HDRS (Hamilton depresión rating Scale) y que los criterios de remisión se alcanzaron en el 20\% de los pacientes a los 6 meses ${ }^{76}$. Los electrodos fueron implantados con los contactos ventrales en el VS y los dorsales en la CI.

El NAcc también se ha considerado como diana en pacientes con depresión refractaria a tratamientos convencionales. Inicialmente, y en un estudio piloto con tres pacientes, se observó un beneficio significativo cuando se aplicó la escala HDRS y en relación con las fases de estimulación "on-off" ha publicado 10 pacientes, de los cuales 5 alcanzaron un $50 \%$ de reducción de dicha escala. En los pacientes con respuesta clínica, la 18F-2-deoxy-glucosa PET mostró que la estimulación del NAcc reduce el metabolismo de la región subgenual y región prefrontal ${ }^{19}$. Según los autores, el electrodo cuadripolar es introducido con el contacto más distal en el centro del NAcc, el contacto 1 en la parte periférica del mismo núcleo y en la región ventral y dorsal de la cápsula estarían colocados los contactos más dorsales ${ }^{97}$.

Sin embargo, desde el análisis anatómico y neuroquirúrgico cuando comparamos las dianas quirúrgicas de los grupos que realizan estimulación en el Nacc y los que van al VS podemos considerar que son idénticas. El grupo de Schlaepfer y Bewernick elige como diana el Nacc, con las siguientes coordenadas (X: 6-7 mm lateral, Y: 1-2 mm anterior a la comisura anterior (CA); Z: 3-4 mm ventral a la misma), mientras que Malone y cols, van al VS y sus coordenadas son $X: 7.5 \mathrm{~mm}$ lateral, Y: $1.5 \mathrm{~mm}$ anterior a la CA y Z: $4 \mathrm{~mm}$ ventral a la misma ${ }^{19,76,97}$. Por lo tanto, podemos consider que la diana estimulada es la misma. 
Tabla 3

Estudios de DBS para depresión refractaria

\begin{tabular}{|c|c|c|c|c|c|c|}
\hline Autor & $\begin{array}{c}\mathrm{N}^{\circ} \\
\text { pacientes }\end{array}$ & $\begin{array}{c}\text { Diana } \\
\text { quirúrgica }\end{array}$ & $\begin{array}{l}\text { Seguimiento } \\
\text { (meses ) }\end{array}$ & $\begin{array}{l}\text { Respuesta } \\
\text { clínica }\end{array}$ & Electrodos & Comentarios \\
\hline $\begin{array}{l}\text { Mayberg y } \\
\text { cols. } 2005^{79}\end{array}$ & 6 & $\begin{array}{l}\text { Area subgenual } \\
\quad \text { (A } 25 \mathrm{~B} \text { ) }\end{array}$ & 6 & $\begin{array}{l}\text { HDRS } \\
\text { 4/6respondedores } \\
\text { 2/6 remisión }\end{array}$ & 3387 & $\begin{array}{l}\text { Confirmación del modelo } \\
\text { propuesto de depresión } \\
\text { reduciendo la actividad } \\
\text { del área subgenual }\end{array}$ \\
\hline $\begin{array}{l}\text { Lozano y cols. } \\
\qquad 2008^{71}\end{array}$ & 20 & $\begin{array}{l}\text { Area subgenual } \\
\qquad \text { (A } 25 \text { B) }\end{array}$ & 12 & $\begin{array}{l}\text { HDRS }>50 \% \text { reducción } \\
55 \% \text { respondedores } \\
35 \% \text { remisión }\end{array}$ & 3387 & $\begin{array}{l}\text { Extensión de grupo de } \\
\text { Mayberg }\end{array}$ \\
\hline $\begin{array}{l}\text { Neimat y cols. } \\
2008^{82}\end{array}$ & 1 & $\begin{array}{l}\text { Area subgenual } \\
\quad \text { (A } 25 \text { B) }\end{array}$ & 12 & $\begin{array}{l}\text { HDRS } \\
\text { Remisión }\end{array}$ & 3387 & $\begin{array}{l}\text { Paciente con una cingulo- } \\
\text { tomía } 12 \text { meses antes }\end{array}$ \\
\hline $\begin{array}{l}\text { Aouizerate y } \\
\text { cols. } 2004^{8}\end{array}$ & 1 & $\mathrm{VS} / \mathrm{CI}$ & 6 & $\begin{array}{l}\text { Remisión OCD } \\
\text { Depresión y } \\
\text { ansiedad mejoría }\end{array}$ & 3387 & TOC y depresión \\
\hline $\begin{array}{l}\text { Schlaepfer y } \\
\text { cols. } 2008^{97}\end{array}$ & 3 & $\mathrm{NAcc} / \mathrm{CI}$ & 23 & $\begin{array}{l}\text { Doble ciego "on-off" } \\
\text { Mejoria "on"estim. } \\
\text { y empeoramiento } \\
\text { off" estim. }\end{array}$ & 3387 & $\begin{array}{l}\text { Selección del NAcc } \\
\text { basado en su papel } \\
\text { de recompensa }\end{array}$ \\
\hline $\begin{array}{l}\text { Bewernick y } \\
\text { cols. } 2010^{19}\end{array}$ & 10 & $\mathrm{VS} / \mathrm{VC}$ & 12 & $\begin{array}{l}50 \% \text { significativa } \\
\text { reducción en } 50 \% \text { de } \\
\text { pacientes. Mejoria de } \\
\text { ansiedad y anhedonía }\end{array}$ & 3387 & $\begin{array}{l}\text { Contacto 0-1: NAcc } \\
\text { Contacto 2: Ventral IC } \\
\text { Contacto 3: medial IC } \\
\text { Extensión del grupo } \\
\text { de Schlaepfer }\end{array}$ \\
\hline $\begin{array}{l}\text { Malone y } \\
\text { cols. } 2009^{76}\end{array}$ & 15 & $\mathrm{VS} / \mathrm{CI}$ & $\begin{array}{l}\text { 6-4 años } \\
(51)\end{array}$ & $\begin{array}{l}\text { HDRS } \\
40 \% \text { respondedores } 6 \mathrm{~m} \\
53.3 \% \text { en última revisión } \\
\text { remisión } 20 \% 6 \mathrm{~m} \\
40 \% \text { en última revisión }\end{array}$ & 3387 & $\begin{array}{l}\text { Contacto } 0: \mathrm{VS} \\
\text { Contacto 1: VS/VC } \\
\text { Contacto 2-3: IC }\end{array}$ \\
\hline $\begin{array}{l}\text { Jimenez y } \\
\text { cols. } 2005^{59}\end{array}$ & 1 & $\begin{array}{l}\text { Pedúnculo } \\
\text { talámico } \\
\text { inferior }\end{array}$ & 24 & $\begin{array}{l}\text { Remisión doble ciego } \\
\text { on-off" periodos) }\end{array}$ & 3389 & \\
\hline $\begin{array}{l}\text { Kosel y cols. } \\
2006^{63}\end{array}$ & 1 & GPi límbico & 18 & $\begin{array}{l}\text { HDRS } \\
\text { Mejoría 50\% }\end{array}$ & 3387 & $\begin{array}{l}\text { Disquinesias tardías y } \\
\text { depresión mayor }\end{array}$ \\
\hline $\begin{array}{l}\text { Sartorius y } \\
\text { cols. } 2010^{96}\end{array}$ & 1 & $\begin{array}{c}\text { Lateral } \\
\text { habenula }\end{array}$ & 13 & Mejoría & 3389 & $\begin{array}{l}\text { Estimulación de estria } \\
\text { medularis }\end{array}$ \\
\hline
\end{tabular}

En la depresión refractaria, tanto la estimulación del área subgenual como la región VS/NAcc/CI obtendrían beneficios similares según las escalas de evaluación en los pacientes intervenidos, con un 50\% de los mismos respondiendo a la misma. Por tanto, la depresión puede ser mejorada quirúrgicamente desde diferentes dianas, introduciendo cambios en el circuito patológico desde distintos puntos. Por último, se debe comentar que los beneficios son progresivos, ya que las escalas de evaluación continúan mejorando durante el seguimiento clínico de los pacientes.

Se han publicado otras dianas quirúrgicas, con un único paciente y como casos clínicos, para la depresión refractaria al tratamiento médico. Así la estimulación del pedúnculo talámico inferior ${ }^{59}$, del GPi en su porción límbica ${ }^{63}$ y la habénula ${ }^{96}$, donde los autores refieren voltajes de 10.5 Vols, por lo que la mejoría podría deberse a que la estimulación, con tal voltaje, alcance puntos distantes a la propia diana quirúrgica, entre ellos la estría medularis.

\section{Discusión}

\section{Por qué DBS en lugar de lesiones}

Basados en la experiencia quirúrgica previa de los trastornos del movimiento, se está abriendo paso la estimulación cerebral de diferentes estructuras profundas en pacientes con problemas psiquiátricos, que son refractarios a los tratamientos convencionales. El porqué se prefiere colocar un estimulador a realizar una cirugía ablativa es obvio, porque no se realiza una lesión que puede considerarse como irreversible. En la literatura se han publicado buenos resultados con cirugía ablativa, simi- 
lares a los actualmente publicados con estimulación; así capsulotomías para TOC o depresiónes, pero los resultados adversos, tras las lesiones se han podido minimizar y en un porcentaje importante de pacientes se realizaron múltiples procedimientos $^{20,23}$. Además las lesiones son bilaterales y esto siempre puede conllevar un riesgo de complicaciones mayor, sobre todo en el lenguaje y de tipo cognitivo en los pacientes intervenidos. Pero esto no sería el principal motivo para preferir actualmente la DBS; la posibilidad de manipular los parámetros de estimulación, permite poder modular con diferentes volúmenes y alcanzar distintas zonas funcionales dentro de una diana. En segundo lugar, con las técnicas de estimulación tenemos la posibilidad de valoraciones clínicas, cuando el paciente sea estimulado o cuando se suspendan los estímulos, lo cual permite realizar estudios doble ciego aleatorios para valorar con una mayor precisión la respuesta clínica de una determinada diana quirúrgica. Por último, comentar que ciertas dianas no se hubieran contemplado si no es por la estimulación, como la posibilidad de manipular el área subgenual para pacientes con depresión refractaria, ya que en dichas estructuras no se puede realizar una lesión. También la lesión del NST en pacientes con TOC, en la que puede inducirse una corea/ balismo tras la lesión debido a la reducción de la actividad palidal sobre el tálamo tampoco hubiera sido contemplada por su alto riesgo quirúrgico.

\section{Mecanismo de acción de la estimulación}

El mecanismo de acción de la estimulación no es conocido actualmente. Si el circuito de los GB tiene una actividad neuronal anormal o patológica, la estimulación puede inducir una disrupción o ruptura de la misma introduciendo un nuevo patrón de actividad que inhibiría el patológico llevando a una mejora clínica. En las dianas quirúrgicas, tanto para trastornos del movimiento como para problemas psiquiátricos, existen somas neuronales y fibras, por lo que el mecanismo intrínseco de acción se desconoce y el mismo puede ser variable dependiendo de la diana quirúrgica ${ }^{70}$. Conocemos que los axones tienen un umbral más bajo que los somas neuronales y que la estimulación puede inducir a que sus terminales liberen los neurotransmisores que almacenan, bien excitadores o inhibidores, pudiendo de esta manera alterar el circuito patológico o incluso modulando la corteza cerebral, como puede ocurrir al estimular el NST ${ }^{45}$.

No podemos pensar que la estimulación actúe de la misma forma en el NST, GPi o Nacc/CI. Así la estimulación en primates parkinsonianos con voltajes clínicamente efectivos produce cambios en los patrones de descarga del GPe y del $\mathrm{GPi}^{52}$ y la estimulación unilateral del NST en ratas tratadas con 6-OHDA (6-hidroxi-dopamina) induce un incremento de la actividad del ácido glutámico en vez de disminuirlo ${ }^{21,68,122}$. Por tanto, cada diana puede responder de diferente forma a la estimulación aunque el resultado clínico sea similar.

La estimulación a largo plazo induce cambios plásticos en el circuito de los GB pudiendo llegar a inducir cambios metabólicos corticales ${ }^{78,91,92}$. Estos cambios pueden no ser inmediatos sino progresivos, por lo que los pacientes pueden mejorar su sintomatología durante el seguimiento clínico. Esto no ocurre con los pacientes con trastornos del movimiento, en los cuales una vez optimizados los parámetros de estimulación la mejoría se puede considerar como establecida.

Podemos considerar que en los trastornos de los GB de tipo motor, el sistema de estimulación puede ser más simple, ya que la hiperactividad del circuito NST-GPi/SNr puede ser alterada desde el NST o bien desde el GPi, mejorando clínicamente al reducir o inhibir esta actividad patológica, en este sentido podría tratarse de una cirugía predictiva. Sin embargo, esto no ocurre en la patología psiquiátrica, donde el circuito límbico puede ser manipulado desde diferentes dianas con resultados similares, e incluso la misma diana quirúrgica puede mejorar procesos diferentes como la estimulación del VS/Nacc/CI mejora la depresión y el TOC y la estimulación del NST mejora la EP y el TOC.

\section{Dianas quirúrgicas en las enfermedades psiquiátricas}

Actualmente hay más de 10 dianas quirúrgicas para las patologías psiquiátricas comentadas, ¿cómo puede existir algo tan poco específico y selectivo? Si quitamos los casos concretos publicados como "case report" en los que un único paciente ha sido intervenido, quedarían dos dianas para cada entidad clínica. Para el TOC, las dianas serían: VS/Nacc/CI y NST. Para la depresión refractaria sería el área subgenual (área 25 de Brodmann) y la región VS/NAcc. Para el Guille de la Tourette la diana sería el Voi y el CM/Pfs del tálamo y el GPi2,19,43,66,71,75,76,79,97,100,107,113. La actuación sobre cada una de estas dianas, induce cambios corticales evaluados en PET, así en los pacientes con TOC que son intervenidos con buena respuesta clínica, la hiperactividad del COF y del área cingular muestra una reducción ${ }^{91,92}$ y los pacientes con depresión con reducción en la escala de Hamilton tienen un hipoactividad del área subgenual $^{78}$. Por lo tanto, el mecanismo de actuación puede ser la modulación de la corteza frontal o bien del circuito límbico, desde los GB en sus porciones límbicas en distintos puntos. Esto podría explicar el mecanismo de mejora de estos pacientes desde las distintas dianas quirúrgicas.

Actualmente no conocemos, por falta de un número de pacientes adecuado, cuál es la diana óptima para cada problema ni para una patología determinada.

Se pueden inducir cambios corticales desde actuacio- 
nes en distintos puntos de la red o circuito funcional, que producen beneficios clínicos tanto en depresión refractaria, como en el TOC o en la enfermedad de Tourette.

Con el mismo concepto, tampoco conocemos, aunque nos centremos en la misma diana, qué pacientes responderán bien al tratamiento y quienes no lo harán, debido a la variabilidad de los diagnósticos clínicos y de la colocación de los electrodos ${ }^{51}$.

Pueden existir diferentes subgrupos de pacientes dentro de cada entidad clínica, lo cual implica que las respuestas pueden ser diferentes. En la actualidad se está dedicando una atención específica a este problema, para identificar a los que se puedan beneficiar de este tratamiento quirúrgico. Esto abre un futuro a estudios clínicos, que ahora están en sus comienzos. Actualmente no existe ningún estudio doble ciego en ninguna de las patologías referidas que compare resultados entre las distintas dianas quirúrgicas.

La DBS para problemas psiquiátricos lleva más de 10 años desde que se publicó el tratamiento con TOC con DBS-CI y para Tourette la DBS-Voi-CM/Pf talámica, ambos en $1999^{84,110}$, sin embargo actualmente no se están interviniendo tantos pacientes como para los trastornos del movimiento. Esto puede ser debido a que los resultados no son tan espectaculares como en los previos $(50 \%$ de pacientes respondedores a los 12 meses y un 30\% considerados en remisión) mientras que en la EP, si el paciente es candidato y el electrodo está en la diana quirúrgica, la cirugía es predictiva y en el temblor, si el electrodo está bien colocado en el Vim, el signo clínico desaparece. Esto no ocurre como hemos visto con la patología psiquiátrica. Todo ello lleva a que todavía se intervengan pocos casos de pacientes con trastornos psiquiátricos y la respuesta clínica al tratamiento quirúrgico pueda variar dependiendo de los casos y actualmente esté siendo estudiada por los distintos grupos involucrados en este tipo de terapia.

\section{Conclusiones}

Los pacientes con trastornos psiquiátricos como TOC, depresión y Gilles de la Tourette, refractarios a tratamientos convencionales, pueden ser intervenidos quirúrgicamente con estimulación cerebral profunda con una reducción significativa de las escalas de evaluación y un beneficio clínico. Un porcentaje de pacientes se puede considerar como "curados" o en remisión, ya que no precisan continuar con medicación y pueden volver a sus actividades diarias.

Con los conocimientos que actualmente tenemos, podemos decir que las patologías como la depresión, TOC y SGT se deben a una alteración del circuito límbico córticoestriado-tálamo-cortical.

Actualmente no existe ninguna diana superior a otras para tratar quirúrgicamente estos trastornos psiquiátricos y no conocemos ningún estudio doble aleatorio entre dife- rentes dianas, en ninguna de las patologías. Por otro lado y con el mismo concepto una diana puede mejorar diferentes patologías. El circuito patológico de los GB podría ser interrumpido desde diferentes puntos o dianas influyendo sobre diferentes áreas corticales.

No conocemos qué pacientes van a responder y quienes no responderán al tratamiento quirúrgico con estimulación, ya que pueden tratarse de subgrupos clínicos dentro de una misma patología diagnóstica.

En un futuro, tanto los procedimientos de imagen, PET previo a la cirugía, colocación de electrodos en dianas determinadas con análisis de tractografía podrían ayudar en este campo.

\section{Bibliografía}

1. Abelson, J., Curtis, G., Sagher, O., Albucher, R., Harrigan, M., Taylor, S., et al.: Deep brain stimulation for refractory obsessive-compulsive disorder. Biol Psychiatry 2005; 57: 510-516.

2. Ackermans, L., Temel, Y., Cath, D., van der Linden, Bruggeman, R., Kleijer, M., et al.: Deep brain stimulation in Tourette syndrome: two targets? Mov Disord 2006; 21: 709713.

3. Agid, Y., Arnulf, I., Bejjani, P., Bloch, F., Bonnet, A.M., Damier, P., et al.: Parkinson's disease is a neuropsychiatric disorder. Adv Neurol 2003; 91: 365-370.

4. Albin, R.L., Mink, J.W.: Recent advances in Tourette syndrome research. Trends in Neurosci 2006; 29: 175-182.

5. Alexander, G.E., Crutcher, M.D., DeLong, M.R.: Basal ganglia-thalamocortical circuits: parallel substrates fore motor, oculomotor, "prefrontal" and "limbic" functions. Prog Brain Res 1990; 85: 119-146.

6. Americal Psychiatric Association AM: Practice guideline for the treatment of patients with major depressive disorders (Revision). Am J Psychiatry 2000; 157: 1-45.

7. Anderson, D., Ahmed, A.: Treatment of patients with intratable obsessive-compulsive disorder with anterior capsular stimulation. J Neurosurg 2003; 98: 1104-1108.

8. Aouizerate, B., Cuny, E., Martin-Guehl, C., Guehl, D., Amieva, H., Benazzouz, A., et al.: Deep brain stimulation of the ventral caudate nucleus in the treatment of obsessive-compulsive disorder and major depression. J Neurosurg 2004; 101 : 682-686.

9. Ardouin, C., Voon, V., Worbe, Y.: Pathological gambling in Parkinson's disease improves on chronic subthalamic nucleus stimulation. Mov Disord 2006; 21: 1941-1946.

10. Aziz, T.Z., Peggs, D., Sambrook, M.A., Crossman, A.R.: Lesion of the subthalamic nucleus for the alleviation of MPTP-induced parkinsonism in the primate. Mov Disord 1991; 6: 288-293.

11. Bajwa, R.J., de Lotbiniére, A., King, R., Jabbari, B., Quatrano, S., Kunze, K., et al.: Deep brain stimulation in 
Tourette's syndrome. Mov Disord 2007; 22: 1346-1350.

12. Ballanger, B., van Eimeren, T., Moro, E., Lozano, A.M., Hamani, C., Boulinguez, P. et al.: Stimulation of the subthalamic nucleus and impulsivity: release your horses. Ann Neurol 2009; 66: 817-824.

13. Baunez, C., Nieoullon, A., Amalric, M.: In a rat model of parkinsonism, lesions of the subthalamic nucleus reverse increases of reaction time but induce a dramatic premature responding deficit. J Neurosci 1995; 15: 6531-6541.

14. Baunez, C., Robbins, T.W.: Bilateral lesions of the subthalamic nucleus induce multiple deficits in an attentional task in rats. Eur J Neurosci 1997; 9: 2086-2099.

15. Bejjani, B.P., Houeto, J.L., Hariz, M., Yelnik, J., Mesnage, V., Bonnet, A.M., et al.: Aggressive behavior induced by intraoperative stimulation in the triangle of Sano. Neurology 2002; 59: 1425-1427.

16. Benazzouz, A., Gross, C., Feger, J., Boraud, T., Bioulac, B.: Reversal of rigidity and improvement in motor performance by subthalamic nucleus high-frequency stimulation in MPTP-treated monkeys. Eur J Neurosci 1993; 5: 382-389.

17. Bergman, H., Wichmann, T., DeLong, M.R.: Reversal of experimental parkinsonism by lesions of the subthalamic nucleus. Science 1990; 249: 1436-1438.

18. Bergman, H., Wichmann, T., Karmon, B., DeLong, M.R.: The primate subthalamic nucleus. II. Neuronal activity in the MPTP model of parkinsonism. J Neurophysiol 1994; 72: 507-519.

19. Bewernick, B., Hurlemann, R., Matussch, A., Kayser, S., Grubert, C., Hadrysiewicz, B., et al.: Nucleus accumbens deep brain stimulation decreases ratings of depression and anxiety in treatment-resistant depression. Biol Psychiatry 2010; 67: 110-116.

20. Bjarkam, C.R., Sorensen, J.C.: Psychosurgery. A historical perspective. Lozano AM, Gildenberg P, Tasker RR (ed). En Textbook of Stereotactic and Functional Neurosurgery $\left(2^{\circ}\right.$ Edición) Springer. 2009. Pp 2867-2886.

21. Bruet, N., Windels, F., Carcenac, C., Feuerstein, C., Bertrand, A., Poupard, A., et al.: Neurochemical mechanism induced by high frequency stimulation of the subthalamic nucleus: increase of extracellular striatal glutamate and GABA in normal and hemiparkinsonian rats. J Neuropathol Exp Neurol 2003; 62: 1228-1240.

22. Burdick, A., Foote, K., Goodman, W., Ward, H., Ricciuti, N., Murphy, T., et al.: Lack of benefit of accumbens/ capsular deep brain stimulation in a patient with both tics and obsessive-compulsive disorder. Neurocase 2010; 1-10.

23. Christensen, D., Laitinen, L.V., Schmidt, L., Hariz, M.: Anterior capsulotomy for treatment of refractory obsessive-compulsive disorder: results in a young and old patient. Stereotact Funct Neurosurg 2002; 79: 234-244.

24. Crossman, A.R., Sambrook, M.A., Jackson, A.: Experimental hemichorea/hemiballismus in the monkey: studies on the intracerebral site of action in a drug-induced dyskinesias.
Brain 1984; 107: 579-596.

25. Darbaky, Y., Forni, C., Amalric, M., Baunez, C.: High frequency stimulation of the subthalamic nucleus has benefitial antiparkinsonian effects on motor function in rats, but less afficiency in a choice reaction time task. Eur J Neurosci 2003; 18: 951-956.

26. Dehning, S., Mehrkens, J.H., Müller, N., Bötzel, K.: Therapy-refractory Tourette syndrome: benefitial outcome with globus pallidus internus deep brain stimulation. Mov Disord 2008; 23: 1300-1302.

27. DeLong, M.R.: Primate models of movement disorders of the basal ganglia origin. (Review). Trends in Neurosci 1990; 13: 281-285.

28. Diederich, N., Kalteis, K., Stamenkovic, M., Pieri, V., Alesch, F.: Efficent internal pallidal stimulation in Gilles de la Tourette syndrome: a case report. Mov Disord 2005; 20: 14961499.

29. Evans, A., Strafella, A., Wrintraub, D., Stacy, M.: Impulsive and compulsive behaviors in Parkinson's disease. Mov Disord 2009; 24: 1561-1570.

30. Fava, M.: Diagnosis and definition of treatment-resistent depression. Biol Psychiatry 2003; 53: 649-659.

31. Ferry, A.T., Ongur, D., An, X., Price, J.L.: Prefrontal cortical projections to the striatum in macaque monkeys: evidence for an organization related to prefrontal networks. J Comp Neurol 2000; 425: 447-470.

32. Fitzgerald, K.D., Welsh, R.C., Gehring, W.J., Abelson, J.L., Himle, J.A., Liberzon, I., et al.: Error-related hyperactivity of the anterior cingulate cortex in obsessive-compulsive disorder. Biol Psychiatry 2005; 57: 287-294.

33. Flaherty, A., Williams, Z., Amirnovin, R., Kasper, E., Rauch, S., Cosgrove, G.R., et al.: Deep brain stimulation of the anterior internal capsule for the treatment of Tourette syndrome: technical case report. Neurosurgery 2005; 57 (ONS Supple 3) 403.

34. Fontaine, D., Mattei, V., Borg, M., von Langsdorff, D., Magnie, M-N., Chanelet, S., et al.: Effect of subthalamic nucleus stimulation on obsessive-compulsive disorder in a patient with Parkinson's disease. J Neurosurg 2004; 100: 1084-1086.

35. François, C., Grabli, D., McCairn, K., Karachi, J.C., Hirsch, E.C., Féger, J., et al.: Behavioural disorders induced by external globus pallidus dysfunction in primates II. Anatomical study. Brain 2004; 127: 2055-2070.

36. Frank, M., Samanta, J., Moustafa, A., Sherman, S.: Hold your horses: impulsivity, deep brain stimulation, and medication in parkinsonism. Science 2007; 318: 1309-1312.

37. Franzini, A., Messina, G., Gambini, O., Muffatti, R., Scarone, S., Cordella, R., et al.: Deep-brain stimulation of the nucleus accumbens in obsessive-compulsive disorder. Clinical, surgical and electrophysiological considerations in two consecutive patients. Neurol Sci 2010; Supl 1: S87-92.

38. Fuster, J.M.: Anatomy of the prefrontal cortex. Fuster 
(Ed) En The Prefrontal Cortex. Academic Press. Elsevier. 2008. Pp 7-58

39. Gallagher, C., Garell, P., Montgomery, E., Madison, W.I.: Hemi tics and deep brain stimulation. Neurology 2006; 66. E12.

40. Gimenez-Amaya, J.M., McFarland, N.R., de las Heras, S., Haber, S.N.: Organization of the ventral thalamic projections to the ventral striatum in the primate. J Comp Neurol 1995; 27: 127-149.

41. Goodman, W., Foote, K., Greenberg, B., Ricciuti, N., Bauer, R., Ward, H., et al.: Deep brain stimulation for intractable obsessive-compulsive disorder: pilot study using a blinded, staggered-onset design. Biol Psychiatry 2010; 67: 535-542.

42. Greenberg, B.D., Price, L.H., Rauch, S.L., Friehs, G., Noren, G., Malone, D. et al.: Neurosurgery for intratable obsessive-compulsive disorder and depression: critical issues. Neurosurg Clin N Am 2003; 14: 199-212.

43. Greenberg, B., Malone, D., Friehs, G., Rezai, A., Kubu, C., Malloy, P., et al.: Three-year outcomes in deep brain stimulation for highly resistant obsessive-compulsive disorder. Neuropsychopharmacology 2006; 31: 2384-2393.

44. Greenberg, B., Gabriels, L.A., Malone, D.A., Rezai, A.R., Friehs, G.M., Okun, M.S., et al.: Deep brain stimulation of the ventral internal capsule/ventral striatum for obsessive-compulsive disorder: worldwide experience. Molecular Psychiatry 2010; 15: 64-79.

45. Gubellini, P., Salin, P., Kerkerian-Le Goff, L., Baunez, $\mathrm{C}$.: Deep brain stimulation in neurological diseases and expermental models: from molecule to complex behavior. Prog Neurobiol 2009; 89: 79-123.

46. Guridi, J., Herrero, M.T., Luquin, M.R., Guillen, J., Ruberg, M., Laguna, J., et al.: Subthalamotomy in parkinsonian monkeys. Behavioural and biochemical analysis. Brain 1996; 119: 1717-1727.

47. Haber, S.N., Kunishio, K., Mizobuchi, M., Lynd-Balta, E.: The orbital and medial prefrontal cuircuit through the primate basal ganglia. J Neurosci 1995; 15: 4851-4867.

48. Haber, S.N., McFarland, N.R.: The concept of ventral striatum in nonhuman primates. Ann N Y Acad Sci 1999; 29; 877: 33-48.

49. Hälbig, T.D., Tse, W., Frisina, P.G., Baker, B.R., Hollander, E., Shapiro, H., et al: Subthalamic deep brain stimulation and impulse control in Parkinson's disease. European J Neurol 2009; 16: 493-497.

50. Hamani, C., Richter, E., Schwalb, J., Lozano, A.M.: Bilateral subthalamic nucleus stimulation for Parkinson's disease: a systematic review of the clinical literature. Neurosurgery 2005; 56: 1313-1324.

51. Hamani, C., Mayberg, H., Snyder, B., Giacobbe, P., Kennedy, S., Lozano, A.M.: Deep brain stimulation of the subcallosal cingulate gyrus for depression: anatomical location of active contacts in clinical responders and a suggested guideline for targeting. J Neurosurg 2009; 111: 1209-1215.

52. Hashimoto, T., Elder, C.M., Okun, M.S., Patrick, S.K., Vitek, J.L.: Stimulation of the subthalamic nucleus changes the firing pattern of pallidal neurons. J Neurosci 2003; 23: 1916-1923.

53. Hassler, R., Dieckmann, G.: Traitement stéréotaxique des tics et cris inarticulés ou coprolaliques considérés comme phénoméne de obsession motrice au cours de la maladie de Guilles de la Tourette. Revue Neurolog. (Paris) 1970; 123: 80100 .

54. Houeto, J.L., Karachi, C., Mallet, L., Pillon, B., Yelnik, Y., Mesnage, V., et al.: Tourette's syndrome and deep brain stimulation. J Neurol Neurosurg Psychiatry 2005; 76: 992-995.

55. Houeto, J.L., Mesnage, V., Mallet, L., Pillo. B., Gergiolo, M., du Moncel, ST., et al,: Behavioural disorders, Parkinson's disease and subthalamic stimulation. J Neurol Neurosurg Psychiatry 2002; 72: 701-707.

56. Huff, W., Lenartz, D., Shormann, M., Sun-Hee, L., Kuhn, J., Koulousakis, A., et al.: Unilateral deep brain stimulation of the nucleus accumbens in patients with teatmentresistant obsessive-compulsive disorder: Outcomes after one year. Clin Neurol Neurosurg 2010; 112: 137-143.

57. Hurwitz, T.A., Mandat, T., Forster, B., Honey, C.: Tract identification by novel MRI signal changes following stereotactic anterior capsulotomy. Stereotact Funct Neurosurg 2006; 84: $228-235$.

58. Jankovic, J.: Tourette's syndrome. N Engl J Med 2001; 345: 1184-1192.

59. Jimenez, F., Velasco, F., Salin-Pascual, R., Henandez, J., Velasco, M., Criales, J., et al.: A patient with a resistant major depression disorder treated with deep brain stimulation in the inferior thalamic peduncle. Neurosurgery 2005; 57: 585-593.

60. Jimenez-Ponce, F., Velasco-Campos, F., Castro-Farfán, G., Nicolini, H., Velasco, A., Salin-Pascual, R., et al.: Preliminary study in patients with obsessive-compulsive disorder treated with electrical stimulation in the inferior thalamic peduncle. Neurosurgery 2009; 65: 203-209.

61. Karachi, C., Grabli, D., Baup, N., Moubayer, S., Tendé, D., François, C., et al.: Dysfunction of the subthalamic nucleus induces behavioral and movement disorders in monkeys. Mov Disord 2009; 24: 1183-1192.

62. Knobel, D., Aybek, S., Pollo, C.: Rapid resolution of dopamine dysregulation syndrome (DDS) after subthalamic DBS for Parkinson's disease (PD): a case report. Cognitive Behavior Neurol 2008; 21: 187-189.

63. Kosel, M., Sturm, V., Frick, C., Lenartz, D., Zeidler, G., Brodesser, D., et al.: Mood improvement after deep brain stimulation of the internal globus pallidus for tardive dyskinesia in a patient suffering from major depression. J Psychiatr Res 2007; 41: 801-803.

64. Krack, P., Batir, A., Van Blercom, N., Chabardes, S., Fraix, V., Ardouin, C., et al.: Five-year follow-up of bila- 
teral stimulation of the subthalamic nucleus in advanced Parkinson's disease. N Engl J Med 2003; 349: 1925-1934.

65. Krack, P., Kumar, R., Ardouin, C., Dowsey, P.L., McVicker, J.M., Benabid, A.L., et al.: Mirthful laughter induced by subthalamic nucleus stimulation. Mov Disord 2001; 16: 867-875.

66. Kuhn, J., Lenartz, D., May, J., Hoff, W., Lee, S., Koulousakis, A. et al.: Deep brain stimulation of the nucleus accumbens and the internal capsule in therapeutically refractory Tourette syndrome. J Neurol 2007; 254: 963-965.

67. Kulisevky, J., Berthier, M.L., Gironell, A., PascualSedano, B., Molet, J., Pares, P.: Mania following deep brain stimulation for Parkinson's disease. Neurology 2002; 59: 1421-1424.

68. Lee, K.H., Kristic, K., Van Hoff: High frequency stimulation of the subthalamic nucleus increases glutamate in the subthalamic nucleus in rats as demonstrated by in vivo enzime-linked glutamate sensor. Brain Res 2007; 1162: 121129.

69. Lipsman, N., Neimat, J.S., Lozano, A.M.: Deep brain stimulation for treatment refractory obsessive-compulsive disorder: the search for a valid target. Neurosurgery 2007; 61: $1-13$.

70. Lozano, A.M., Dostrovsky, J., Chen, R., Ashby, P.: Deep brain stimulation for Parkinson's disease: disrupting the disruption. Lancet Neurology 2002; 1: 225-231.

71. Lozano, A.M., Mayberg, H.S., Giacobbe, P., Hamani, C., Craddock, C., Kennedy, S.: Subcallosal cingulate gyrus deep brain stimulation for treatment-resistent depression. Biol Psychiatry 2008; 64: 461-467.

72. Lu, C., Bharmal, A., Suchowersky, O.: Gambling and Parkinson disease: Arch Neurol 2006; 63: 298.

73. Maciunas, R.J., Maddux, B., Riley, D., Whitney, C., Schoenberg, M., Ogrocki, P., et al.: Prospective randomized double-blind trial of bilateral thalamic deep brain stimulation in adults with Tourette syndrome. J Neurosurg 2007; 107: 1004-1014.

74. Mallet, L., Mesnage, V., Houeto, J-L., Pelissolo, A., Yelnik, Y., Behar, C., et al.: Compulsions, Parkinson's disease and stimulation. Lancet 2002; 360: 1302-1304.

75. Mallet, L., Polosan, M., Jaafari, N., Baup, N., Welter, M-L., Fontaine, D., et al.: Subthalamic nucleus stimulation in severe obsessive-compulsive disorder. New Engl J Med 2008; 359: 2121-2134.

76. Malone, D., Dougherty, D., Rezai, A., Carpenter, L., Fiehs, G., Eskandar, E., et al.: Deep brain stimulation of the ventral capsule/ventral striatum for treatment-resistant depression. Biol Psychiatry 2009; 65: 267-275.

77. Martinez-Torres, I., Hariz, M., Zrinzo, L., Foltynie, T., Limousin, P.: Improvement of tics after subthalamic nucleus deep brain stimulation. Neurology 2009; 72: 1787-1789.

78. Mayerg, H.S.: Limbic-cortical dysregulation: a proposed model of depression. J Neuropsychiatry Clin Neurosci
1997; 9: 471-481.

79. Mayberg, H.S., Lozano, A.M., Voon, V., McNeely, E., Seminowicz, D., Hamani, C., et al.: Deep brain stimulation for treatment-resistant depression. Neuron 2005; 45: 651-660.

80. Nakano, K.: Neural circuits and topographic organization of the basal ganglia and related regions. Brain Dev 2000; 22 (Supple 1) 5-16.

81. Nambu, A., Takada, M., Inase, M., Tokuno, H.: Dual somatotopical representations in the primate subthalamic nucleus: evidence for ordered but reversed body-map trnsformations from the primary motor cortex and the supplementary motor area. J Neurosci 1996; 16: 2671-2683.

82. Neimat, J., Hamani, C., Giacobbe, P., Merskey, H., Kennedy, S., Mayberg, H., et al.: Neural stimulation successfully treats depression in patients with prior ablative cingulotomy. Am J Psychiatry 2008; 165: 687-693.

83. Neuner, I., Podoll, K., Lenartz, D., Strum, V., Schneider, F.: Deep brain stimulation in the nucleus accumbens for intratable Tourette's syndrome: follow-up report of 36 months. Biol Psychiatry 2009; 65: e5-e6.

84. Nuttin, B., Cosyns, P., Demeulenmeester, H., Gybels, J., Meyerson, B.: Electrical stimulation in anterior limbs of internal capsules in patients with obsessive-compulsive disorder. Lancet 1999; 354: 1526.

85. Nuttin, B., Gabriels, L., Cosyns, P., Meyerson, B., Andreewitch, S., Sunaert, S., et al.: Long-term electrical capsular stimulation in patients with obsessive-compulsive disorder. Neurosurgery 2003; 52: 1263-1274.

86. Okun, M.S., Fernandez, H., Foote, K., Murphy, T., Goodman, W.: Avoiding deep brain stimulation failures in Tourette syndrome. J Neurol Neurosurg Psychiatry 2008; 79: 111-112.

87. Ostergaard, K., Sunde, N.: Evolution of Parkinson's disease during 4 years of bilateral deep brain stimulation of the subthalamic nucleus. Mov Disord 2006; 21: 624-631.

88. Pallanti, S., Quercioli, L.: Treatment refractory obsessive-compulsive disorder: methological issues, operational definitions and therapeutic lines. Prog Neuropsychopharmacol Biol Psychiatry 2006; 30: 400-412.

89. Parent, A., Hazrati, L.N.: Functional anatomy of the basal ganglia. I. The cortico-basal ganglia-thalamo-cortical looop. Brain Res Rev 1995; 20: 91-127.

90. Peterson, B.S.: Neuroimaging studies of Tourette synndrome: a decade of progress. Cohen DJ, Jankovik J, Goetz CG (Ed). En Tourette syndrome. Vol 85. Advances in Neurology. Philadelphia. Lippincott Williams and Wilkins.2001; Pp 179196.

91. Rauch, S.L.: Neuroimaging and neurocircuity models pertaining to neurosurgical treatment of psychiatric disorders. Neurosurg Clin N Am 2003; 14: 213-223.

92. Rauch, S.L., Dogherty, D.D., Malone, D., Rezai, A., Friehs, G., Fischman, A.J., et al.: A functional neuroimaging investigation of deep brain stimulation in patients with obses- 
sive-compulsive disorder. J Neurosurg 2006; 104: 558-565.

93. Rickards, H., Wood, C., Cavanna, A.: Hassler and Dieckmann's seminal paper on stereotactic thalamotomy for Gilles de la Tourette syndrome: translation and critical reappraisal. Historical review. Mov Disord 2008; 23: 19661972.

94. Rodriguez-Oroz, M.C., Obeso, J.A., Lang, A.E., Houeto, J.L., Pollak, P., Rehncrona, S., et al.: Bilateral deep brain stimulation in Parkinson's disease: a multicentre study with 4 years follow-up. Brain 2005; 128: 2240-2249.

95. Saint-Cyr, J.A., Trepanier, L., Kumar, R., Lozano, A.M.: Neuropsycological consequences of chronic bilateral stimulation of the subthalamic nucleus in Parkinson's disease. Brain 2000; 123: 2091-2108.

96. Sartorius, A., Kiening, K., Kirsch, P., von Gall, C., Unterberg, A., Henn, F., et al.. Remission of major depression under deep brain stimulation of the lateral habenula in a therapy-refractory patient. Biol Psychiatry 2010; 67: e 9-e 11.

97. Schlaepfer, T., Cohen, M., Frick, C., Kosel, M., Brodesser, D., Axmacher, N., et al.: Deep brain stimulation to reward circuitry alleviates anhedonia in refractory major depression. Neuropsychopharmacology 2008; 33: 368-377.

98. Schüpbach, W.M.M., Chastan, N., Welter, M.L., Houeto, J.L., Masnage, V., Bonnet, A.M., et al,: Stimulation of the subthalamic nucleus in Parkinson's disease: a 5 year follow up. J Neurol Neurosurg Psychiatry 2005; 76: 16401644.

99. Seminowicz, D.A., Mayberg, H.S., McIntosh, A.R., Golapple, K.K., Kennedy, S., Segal, Z., et al.: Limbic-frontal circuitry in Major Depression: a path modeling metanalysis. Neuroimage 2004; 22: 409-418.

100. Servello, D., Porta, M., Sassi, M., Brambilla, A., Robertson, M.M.: Deep brain stimulation in 18 patients with severe Gilles de la Tourette syndrome refractory to treatment: the surgery and stimulation. J Neurol Neurosurg Psychiatry 2008; 79: 136-142.

101. Shahed, J., Poysky, J., Kenney, C., Simpsom, R., Jankovic, J.: GPi deep brain stimulation for Tourette syndrome improves tics and psychiatric comorbities. Neurology 2007; 68: 159-161.

102. Shields, D., Cheng, M.L., Flaherty, A., Gale, J., Eskandar, E.N.: Microelectrode-guided deep brain stimulation for Tourette syndrome: within-subject comparison of different stimulation sites. Stereotact Funct Neurosurg 2008; 86: 8791.

103. Schupbach, M., Gargiulo, M., Welter, M.L.: Neurosurgery in Parkinson disease: a distressed mind in a repaired body? Neurology 2006; 66: 1811-1816.

104. Smeding, H.M., Goudriaan, A.E., Foncke, E.M.: Pathological gambling after bilateral subthalamic nucleus stimulation in Parkinson's disease. J Neurol Neurosug Psychiatry 2007; 78: 517-519.

105. Spiegel, E.A., Wycis, H.T., Marks ,M,. Lee, A.S.:
Stereotaxic apparatus for operations in human brain. Science 1947; 108: 349-350.

106. Stein, D.J.: Obsessive-compulsive disorder. Lancet 2002; 360: 397-405.

107. Sturm, V., Lenartz, D., Koulouskis, A., Treuer, H., Herlhoz, K., Klein, J., et al.: The nucleus accumbens: a target for deep brain stimulation in obsessive-compulsive and anxiety disorders. J Chem Neuroanatomy 2003; 26: 293299.

108. Tan, S.K., Temel, Y., Blokland, A., Steinbusch, H., Visser-Vanderwalle, V.: The subthalamic nucleus: from response selection to execution. J Che Neuroanatomy 2006; 31 : 155-161.

109. Ulla, M., Thobois, S., Lamaire, J.J., Schmitt, A., Deronst, P., Broussolles, E., et al.: Manic behaviour induced by deep brain stimulation in Parkinson's disease: evidence of substantia nigra implication? J Neurol Neurosurg Psychiatry 2006; 77: 1363-1366.

110. Vandewalle, V., van der Linden, C., Groenewegen, H.J., Caemaert, J.: Stereotactic treatment of Gilles de la Tourette syndrome by high frequency stimulation of the thalamus. The Lancet 1999; 353: 724.

111. Vernaleken, I, Kuhn. J., Lenartz, D., Raptis, M., Huff, W., Janouschek, H., et al.: Bithalamic deep brain stimulation in Tourette syndrome is associated with reduction in dopaminergic transmission. Biol Psychiatry 2009; 66: e15-e17.

112. Vilela-Fhilo, O.: Bilateral globus pallidus externus deep brain stimulation (GPe-DBS) for the treatment of Tourette syndrome: an ongoing prospective controlled study (Abstract). Stereotact Funct Neurosurg 2007; 85: 42-43.

113. Visser-Vandewalle, V., Temel, Y., Boon, P., Vreeling, F., Colle, H., Hoogland, G., et al.: Chronic bilateral thalamic stimulation: a new therapeutic approach in intratable Tourette syndrome. J Neurosurg 2003; 99: 1094-1100.

114. Visser-Vanderwalle, V., van der Linden, C., Temel, Y. Celik, H., Ackermans, L., Spincemaille, G. : Long-term of bilateral subthalamic nucleus stimulation in advanced Parkinson's disease: a four year follow-up study. Parkinsonism Relat Disord 2005; 11: 157-165.

115. Voon, V., Saint-Cyr, J., Lozano, A.M., Moro, E., Poon, Y.Y., et al.: Psychiatric symptoms in patients with Parkinson disease presenting for deep brain stimulation surgery. J Neurosurg 2005; 103: 246-251.

116. Voon, V., Kubu, C., Krack, P., Houeto, J-L., Tröster, A.: Deep brain stimulation: neuropsychological and neuropsychiatric issues. Mov Disord 2006; 21 (Supl 14) 305-327.

117. Voon, V., Potenza, M.N., Thompsen, T.: Medication related impulse control and repetitive bahaviors in Parkinson's disease. Current Opinion in Neurology 2007; 20: 484-492.

118. Voon, V., Thompsen, T., Miyasaky, J.M. y cols.: Factors associated with dopaminergic drug-related pathological gambling in Parkinson's disease. Arch Neurol 2007; 64: 212- 
216.

119. Voon, V., Krack, P., Lang, A.E., Lozano, A.M., Dujardin, K., Shüppbach, M., et al.: Parkinson's disease, DBS and suicide: a role for serotonin? Brain 2009 Jun 24

120. Weintraub, D., Siderowf, A.D., Potenza, M.N., et al.: Associated of dopamine agonist use with impulse control disorders in Parkinson's disease. Arch Neurol 2006; 63: 969973.

121. Welter, M-L., Mallet, L., Houeto, J.L., Karachi, C., Czernecki, V., Cornu, P., et al.: Internal pallidal and thalamic stimulation in patients with Tourette syndrome. Arch Neurol 2008; 65: 952-957.

122. Windels, F., Carnenac, C., Poupard, A., Savasta, M.: Pallidal origin of GABA release within the substantia nigra pars reticulata during high-frequency stimulation of the subthalamic nucleus. J Neurosci 2005; 18: 5079-5086.

123. Wojtecki, L., Nickel, J., Timmermann, L., Maaruof,
2011; 22: 5-22

M., Südmeyer, M., Schneider, F., et al.: Pathological crying induced by deep brain stimulation. Mov Disord 2007; 15: 1314-1316.

124. Worbe, Y., Baup, N., Grabli, D., Chaigneau, M., Mounayer, S., McCairn, K., et al.: Behavioral and movement disorders induced by local inhibititory dysfunction in primate striatum. Cereb Cortex 2009; 19: 1844-1856.

125. Yelnik, J.: Functional anatomy of the Basal Ganglia. Mov Disord 2002; 17 (Supl 3): 15-21.

Guridi, J.; Aldave, G.: Dianas quirúrgicas en el tratamiento de enfermedades psiquiátricas. Desde el movimiento a las emociones.Neurocirugía 2011; 22: 5-22.

Correspondencia. Dr Jorge Guridi. Servicio de Neurocirugía. Clínica Universidad de Navarra. Pio XII sn. 31008 Pamplona jguridi@unav.es 